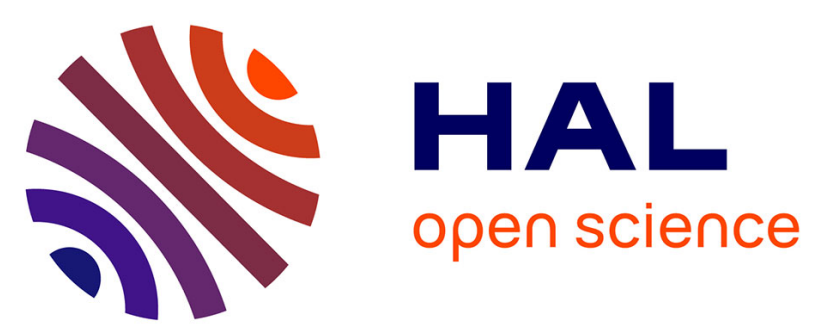

\title{
Failure in accretionary wedges with the maximum strength theorem: numerical algorithm and 2D validation
}

\author{
P. Souloumiac, K. Krabbenhøft, Y. M. Leroy, B. Maillot
}

\section{- To cite this version:}

P. Souloumiac, K. Krabbenhøft, Y. M. Leroy, B. Maillot. Failure in accretionary wedges with the maximum strength theorem: numerical algorithm and 2D validation. Computational Geosciences, 2010, 14 (4), pp.793-811. 10.1007/s10596-010-9184-4 . hal-00584137

HAL Id: hal-00584137

https://hal-mines-paristech.archives-ouvertes.fr/hal-00584137

Submitted on 11 Apr 2011

HAL is a multi-disciplinary open access archive for the deposit and dissemination of scientific research documents, whether they are published or not. The documents may come from teaching and research institutions in France or abroad, or from public or private research centers.
L'archive ouverte pluridisciplinaire HAL, est destinée au dépôt et à la diffusion de documents scientifiques de niveau recherche, publiés ou non, émanant des établissements d'enseignement et de recherche français ou étrangers, des laboratoires publics ou privés. 


\title{
Failure in accretionary wedges with the maximum strength theorem: numerical algorithm and $2 \mathrm{D}$ validation.
}

\author{
P. Souloumiac \\ Laboratoire MSS-Mat, CNRS, \\ Ecole Centrale Paris, Chatenay Malabry, France, \\ K. Krabbenhøft \\ Centre for Geotechnical and Materials Modelling, \\ University of Newcastle, NSW 2308, Australia, \\ Y.M. Leroy \\ Laboratoire de Géologie, CNRS, \\ École Normale Supérieure, Paris, France, \\ and \\ B. Maillot \\ Département Géosciences et Environnement, \\ Université de Cergy-Pontoise, France.
}

\begin{abstract}
The objective is to capture the 3D spatial variation in the failure mode occuring in accretionary wedges, and their analogue experiments in the laboratory, from the sole knowledge of the material strength and the structure geometry. The proposed methodology relies on the maximum strength theorem which is inherited from the kinematics approach of the classical limit analysis. It selects the optimum virtual velocity field which minimizes the tectonic force. These fields are constructed by interpolation thanks to the spatial discretization conducted with ten-noded tetrahedra in 3D, and six-noded triangles in $2 \mathrm{D}$. The resulting, discrete optimization problem is first presented emphasizing the dual formalism found most appropriate in the presence of non-linear strength criteria, such as the Drucker-Prager criterion used in all reported examples.

The numerical scheme is first applied to a perfectly-triangular $2 \mathrm{D}$ wedge. It is known that failure occurs to the back, for topographic slope smaller than, and to the front for slope larger than, a critical slope, defining sub-critical and super-critical slope stability conditions, respectively. The failure mode is characterized by the activation of a ramp, its conjugate back thrust and the partial or complete activation of the décollement. It is shown that the critical slope is captured precisely by the proposed numerical scheme, the ramp and the back thrust corresponding to regions of localized virtual strain. The influence of the back-wall friction on this critical slope is explored. It is found that the failure mechanism is characterized by a thrust rooting at the base of the back wall and the absence of back thrust, for small enough values of the friction angle. This influence is well explained by the Mohr construction and further validated with experimental results with sand, considered as an analogue material. 3D applications of the same methodology are presented in a companion paper.
\end{abstract}

Submitted for publication.

October 26, 2009 


\section{Introduction}

The objective is to determine the 3D failure mode which characterizes the onset of thrusting or folding in fold-and-thrust belts and in accretionary wedges. The numerical method which is proposed has its root in the kinematics approach of limit analysis although only the knowledge of the material strength is required. The numerical algorithm and its $2 \mathrm{D}$ validation are presented in this contribution, the 3D applications in a companion paper (Souloumiac et al., 2009).

The kinematics of 2D folds and thrusts has been studied at length and is now well captured by geometrical constructions inspired by the seminal work of Suppe (1983). The absence of any concept of mechanics, such as material strength and mechanical equilibrium render however impossible the comparison between two geometrical constructions necessary to select the most relevant. The merit of these constructions is however clear in view of their simplicity and their potential application in the oil industry, once completed by the computation of the temperature evolution (Zoetemeijer and Sassi, 1992, Sciamanna et al., 2004).

The line of work which has been followed by the authors tries to take the most advantage of the 2D geometrical construction while accounting for material strength and mechanical equilibrium. The principle of minimum dissipation was applied by Maillot and Leroy (2003) in their study of a simple fault-bend fold, with either brittle or ductile material response, to find the optimum orientation of the back thrust. A more rigorous framework is now adopted, based on the maximum strength theorem for frictional and cohesive materials (Salençon, 1974, 2002). It was applied to the evolution of a kink-fold by Maillot and Leroy (2006) proposing that, at any stage of the structure development, its main geometrical attributes, such as the kink dip and width, could be found by minimizing the upper bound to the applied tectonic force. Cubas and al. (2008) extended this argument to study sequences of thrusts within an accretionary wedge. Souloumiac et al. (2008) proved that the optimum stress state could be calculated at any step of the thrusting sequence development, based on the static approach of the limit analysis.

There is a definite desire to propose 3D constructions of folding and thrusting which is often inhibited by the lack of intuition for parameterizing simply the failure mechanism (e.g. ramp and back thrust system) at the onset and during the development of the fold. It is thus necessary to develop a systematic procedure to study the failure mode of $3 \mathrm{D}$ geological structures. For the onset, the kinematics approach of limit analysis could provide a first insight on the failure mode. It is the subject of the present contribution and it is hoped that the results could help in constructing the 3D kinematics of the evolving structures.

The proposed method, referred to as the maximum strength theorem, is based on the kinematics approach of classical limit analysis. It is emphasized that a complete plasticity theory is not required and the provision for the cohesive and frictional rocks of interest of a strength domain, convex in the stress space, suffices to obtain an upper bound to the applied tectonic force. Over the years, a number of different numerical formulations of the maximum strength (or upper bound) theorem have been proposed. Early formulations, focusing on two-dimensional problems (Anderheggen and Knöpfel, 1972; Pastor, 1978; Bottero et al., 1980; Sloan, 1989), typically involved a linearization of the strength domain and made use of the simplex method or one of its derivatives to solve the resulting linear programs. Inspired by the progress in general convex programming, these linear programming formulations have recently been replaced by more general non-linear formulations avoiding the need to linearize (Lyamin and Sloan, 2002; Krabbenhøft and Damkilde, 2003). The most recent development on this front has been the applications of the so-called conic programming algorithms to solve typical limit analysis problems such as the ones considered here as well as a range of other plasticity problems (Krabbenhøft et al., 2007; Krabbenhøft et al., 2008). These algorithms are particularly suited for dealing with non-smooth strength domains such as those typically characterizing the strength of cohesive, 
frictional materials (Drucker-Prager, Mohr-Coulomb, etc...).

In its primal form the maximum strength theorem is formulated in terms of kinematic variables, the virtual velocities. Their distribution is constructed by interpolation thanks to a space discretization. This primal form with discretization leads to a convex minimization problem. Alternatively, it is possible to work directly with the dual form of the theorem which leads to a maximization problem reminiscent of the static approach leading to lower bounds to the tectonic force. The dual variables of the velocities (of its symmetric gradient to be more precise) in the sense of power are regarded as stresses after appropriate scaling, although they do not constitute statically admissible fields (these dual variables do not satisfy equilibrium). From a numerical point of view, this alternative, dual approach has a number of advantages. For example, it is possible to impose completely general strength criteria in a straightforward manner whereas a primal upper bound formulation would require the specification of the corresponding support function. This function defines the maxium power which could be provided for a given velocity and strength domain. Its analytical expression is certainly non-trivial to derive and the resulting constraints difficult to account for in a classical optimization code. Furthermore, following the approach proposed by (Krabbenhøft et al., 2005), the incorporation of kinematically admissible velocity discontinuities is straightforward and will be proposed in this paper for the general three-dimensional case for the first time.

The paper contents are as follows. The next section is devoted to the presentation of the numerical algorithm. The 2D setting is most suited for such presentation for sake of simplicity and the extension to 3D is postponed to Appendix B. The construction of the dual problem is highlighted with the help of the primal-dual algorithm of linear programming summarized in Appendix A. Appendix C presents the link between these strength domains, typical of soil mechanics, and the conic programming algorithms adopted in Mosek (2008), which is used for all examples reported here. Section 3 is concerned with 2D applications to accretionary wedges of perfect triangular shape. Failure in the bulk occurs either to the back or to the front, with the complete activation of the weak décollement at the base, depending on the topographic slope. The transition from sub-critical (failure to the back) to super-critical (failure to the front) is captured exactly, validating the numerical procedure. It is shown that the friction angle on the back wall influences the failure mode for sub-critical conditions. For small values of the friction angle, a single ramp roots to the base of the back wall whereas a ramp and back thrust occurs for larger values. The transition in failure mode occurs for a friction angle detected numerically which is exactly the one predicted by the Mohr's construction. It is also shown that these two modes of failure are reproduced in the laboratory experiments with sand by selecting the appropriate friction conditions at the back wall contact.

\section{The maximum strength theorem with spatial discretization}

The objective of this section is to present in three steps the theory applied in the next section for $2 \mathrm{D}$ wedges and for $3 \mathrm{D}$ examples in the companion paper. The first step is the presentation of the upper bound theorem of classical limit analysis, as it is found in Salençon (2002) and Maillot and Leroy (2006). It is proposed here to approximate the strength domain externally by a series of hyper-plane, in the appropriate stress space, to facilitate the set up of the optimization problem. The second step is the discretization of the space and the construction of interpolations for the virtual velocities as well as for the virtual scalars associated to these hyper-planes. The third step consists in the dualization of the upper bound problem after discretization, resulting in a maximization problem where the basic unknowns are scaled to have dimension of stress. This dual formulation is used in all examples but should not be confused with the lower 
bound approach (constructed with statically admissible stress fields) for reasons which are also discussed.

\subsection{Summary of the upper bound theorem of limit analysis}

The upper bound theorem of limit analysis is called here the maximum strength theorem to emphasize that only the concept of strength is required. This theorem is now presented in details.

The starting point is the theorem of virtual power which states the equality between the internal and the external powers for any kinematically admissible (KA) velocity field. The set $\mathcal{S}_{u}$ of KA fields comprises any field $\hat{\mathbf{U}}$ which is zero over part of the boundary $\partial \Omega_{u}$ where the displacements are prescribed. Elements of $\mathcal{S}_{u}$ are identified by a superposed hat. The external power, defined by

$$
\mathcal{P}_{\text {ext }}(\hat{\mathbf{U}})=\int_{\Omega} \rho \mathbf{g} \cdot \hat{\mathbf{U}} d V+\alpha \int_{\partial \Omega_{T}} \mathbf{T}^{o} \cdot \hat{\mathbf{U}} d S,
$$

is due to the power of the velocity over the body force $\mathbf{g}, \rho$ is the material density, and of the force applied on part of the boundary $\partial \Omega_{T}$. This applied force is assumed to be known in distribution $\mathbf{T}^{o}$ but not in its intensity defined by the scalar $\alpha$ which is the unknown of the problem and for which we seek the best upper bound. Note that in (1) and in what follows, vectors and subsequently tensors, are identified with bold characters. The internal power is given by

$$
\mathcal{P}_{\text {int }}(\hat{\mathbf{U}})=\int_{\Omega} \boldsymbol{\sigma}: \mathbf{d}(\hat{\mathbf{U}}) d V,
$$

where $\boldsymbol{\sigma}$ and $\mathbf{d}(\hat{\mathbf{U}})$ are the Cauchy stress tensor and the virtual rate of deformation tensor (also denoted $\hat{\mathbf{d}}$ ) based on $\hat{\mathbf{U}}$, respectively. The double dot product in (2) between these two tensors results in $\sigma_{i j} \hat{d}_{j i}$ in terms of their components in an orthonormal basis. The expression (2) for the internal power does not account for potential discontinuities in the velocity fields and bulk deformation is the only source of dissipation. Explicit account of discontinuities, which orientations are part of the unknowns of the problem, is typical of analytical developments but is not necessary in the numerical formulation considered in this paper. However, pre-defined, physical discontinuities thus of known geometry are approached as zones of bulk material having a zero thickness. Their activation is marked by a localized deformation within these narrow zones. The conventional finite-element formulations cannot cope with the limit of zero length in one direction for an element because of the resulting ill-conditioning of the stiffness array (see e.g. Day and Potts, 1994). To the contrary, the formulation adopted in the following does not involve such complication. Indeed, as it will be discussed in the last part of this section, it is entirely possible to include patches of elements with a thickness identically set to zero. This approach was first suggested by Krabbenhøft et al. (2005) in the context of linear velocity elements and is extended here to quadratic velocity elements in $2 \mathrm{D}$ and further generalized to $3 \mathrm{D}$.

Coming back to the internal power (2), note that the stress field is unknown and its elimination is desired. For that purpose, we take advantage of the material maximum strength. The stress is required to remain within the strength domain denoted $G(\boldsymbol{\sigma})$. The strength of cohesive, frictional faults is usually described in terms of the Coulomb criterion and for pristine, bulk materials the strength domain is

$$
G(\boldsymbol{\sigma})=\left\{\boldsymbol{\sigma} \mid \sigma_{I}-\sigma_{I I I}+\left(\sigma_{I}+\sigma_{I I I}\right) \sin \phi-C \cos \phi \leq 0\right\},
$$


where $\sigma_{I}$ and $\sigma_{I I I}$ are the minor and major principal stresses (continuum mechanics convention: tensile stresses are positive, $\left.\sigma_{I} \geq \sigma_{I I I}\right)$ and $C$ and $\phi$ are the cohesion and the friction angle respectively. Failure is described in the $2 \mathrm{D}$ plane which is orthogonal to the intermediate stress direction. The principal stresses could be eliminated in favor of the stress components such that $(3)$ reads in a $2 \mathrm{D}$ setting

$$
\begin{array}{r}
G(\boldsymbol{\sigma})=\left\{\boldsymbol{\sigma} \mid \sigma_{e}+2 P \sin \phi_{B}-2 C \cos \phi \leq 0\right\} \\
\text { with } \quad \sigma_{e}=\sqrt{\left(\sigma_{x x}-\sigma_{y y}\right)^{2}+4 \sigma_{x y}^{2}} \quad, P=\left(\sigma_{x x}+\sigma_{y y}\right) / 2,
\end{array}
$$

in which $\sigma_{e}$ and $P$ are referred to as the equivalent shear stress and the in-plane mean stress, respectively. The determination of the intermediate stress direction becomes a burden in 3D applications and it is more convenient to consider the strength domain bounded by the DruckerPrager criterion:

$$
\begin{array}{r}
G_{D P}(\boldsymbol{\sigma})=\left\{\boldsymbol{\sigma} \mid \alpha_{D P} I_{1}+\sqrt{J_{2}}-C_{D P} \leq 0\right\}, \\
\text { with } \quad I_{1}=\operatorname{tr}(\boldsymbol{\sigma}), \quad \mathrm{J}_{2}=\frac{1}{2} \operatorname{tr}\left(\boldsymbol{\sigma}^{\prime} \cdot \boldsymbol{\sigma}^{\prime}\right), \quad \boldsymbol{\sigma}^{\prime}=\boldsymbol{\sigma}-\frac{1}{3} \operatorname{tr}(\boldsymbol{\sigma}) \boldsymbol{\delta},
\end{array}
$$

in which $I_{1}$ and $J_{2}$ are the first invariant of the stress and the second invariant of the deviatory stress, respectively. Note that $\boldsymbol{\sigma}^{\prime}$ is the deviatory stress and $\boldsymbol{\delta}$ the second-order identity tensor in (5). The two material parameters in (5) are the friction coefficient and the cohesion for the Drucker-Prager criterion and they are conviniently defined as

$$
\alpha_{D P}=\frac{\tan \phi}{\sqrt{9+12 \tan ^{2} \phi}}, \quad C_{D P}=\frac{3 C}{\sqrt{9+12 \tan ^{2} \phi}},
$$

so that the domain boundaries described by (3) and (5) coincide for $2 \mathrm{D}$ plane-strain problems (see e.g. Davis and Selvadurai, 2002, for further details).

Most if not all strength domains considered in the literature are convex. Consequently, the maximum power $\boldsymbol{\sigma}: \hat{\mathbf{d}}$ is bounded and given for a given velocity $\hat{\mathbf{U}}$ by the support function $\pi(\hat{\mathbf{d}})$. It depends on the geometry of the strength domain boundary and of course on the velocity field. A graphical method to construct this function is presented in Figure 1 where stress tensors are represented as vectors. The strength domain has an arbitrary, albeit convex, boundary in the stress space. Superpose in this stress space the virtual rate of deformation $\hat{\mathbf{d}}$ despite the difference in dimension. This virtual rate of deformation is normal to the hyperplane represented with a dashed line. Translate this plane towards the strength domain, as illustrated by the dotted curve, and the point of contact, denoted $\boldsymbol{\sigma}^{*}$, is the stress providing the maximum power according to classical convex analysis. Consequently: $\pi(\hat{\mathbf{d}})=\boldsymbol{\sigma}^{*}: \hat{\mathbf{d}}$ and the selection of $\boldsymbol{\sigma}^{*}$ is indeed a function of the orientation of $\hat{\mathbf{d}}$ and of the shape of the strength domain boundary.

The analysis of the $2 \mathrm{D}$ results in section 3 will be facilitated with the explicit expression of the support function. It reads

$$
\begin{array}{ll}
\text { case } 1: \operatorname{tr}(\hat{\mathbf{d}})>\left(\left|\hat{\mathrm{d}}_{1}\right|+\left|\hat{\mathrm{d}}_{2}\right|\right) \sin \phi, & \pi(\hat{\mathbf{d}})=\frac{C}{\tan \phi} \operatorname{tr}(\hat{\mathbf{d}}), \\
\text { case } 2: \operatorname{tr}(\hat{\mathbf{d}})=\left(\left|\hat{\mathrm{d}}_{1}\right|+\left|\hat{\mathrm{d}}_{2}\right|\right) \sin \phi, & \pi(\hat{\mathbf{d}})=C \cos \phi\left(\left|\hat{d}_{1}\right|+\left|\hat{d}_{2}\right|\right), \\
\text { case } 3: \operatorname{tr}(\hat{\mathbf{d}})<\left(\left|\hat{\mathrm{d}}_{1}\right|+\left|\hat{\mathrm{d}}_{2}\right|\right) \sin \phi, & \pi(\hat{\mathbf{d}})=+\infty,
\end{array}
$$

for bulk materials having the strength limit defined by the Coulomb criterion (3) in 2D (Salençon, 2002). In (7), $\hat{d}_{1}$ and $\hat{d}_{2}$ are the $2 \mathrm{D}$ principal values of the virtual rate of deformation 
tensor. This example reveals that the support function could be infinite for some orientations of the rate of deformation. More specifically, the trace of the virtual rate of deformation has to be positive, for the bound to be finite, implying a virtual dilation which we will not try to interpret physically. This is due to the infinite resistance in pure compression assumed for the Coulomb criterion.

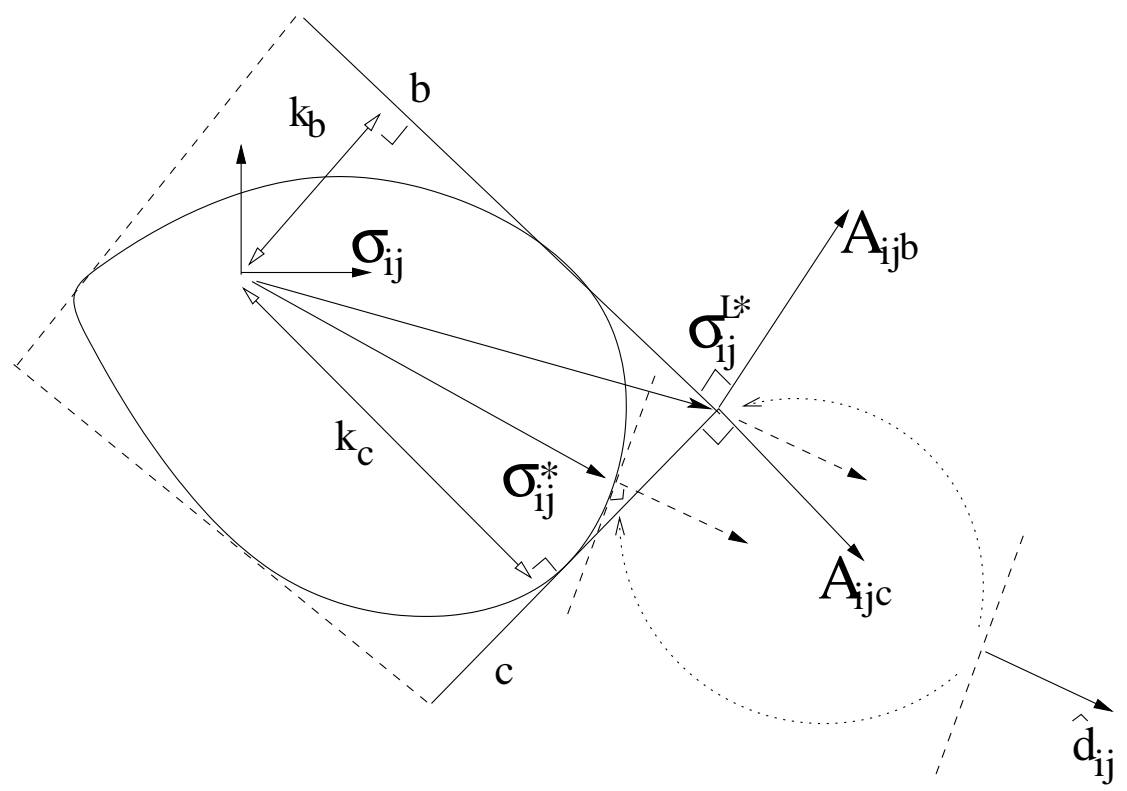

Figure 1: The graphical method to construct the support function for a convex strength domain. The linearized strength domain bounds the support function externally and is represented by four hyper-planes (two dashed and two solid lines).

It is found convenient for what follows to approximate externally the strength domain boundary with a series of $n$ hyper-planes in the stress space. Each plane bounds a half-space defined by

$$
\mathbf{A}_{a}: \boldsymbol{\sigma}-k_{a} \leq 0, \quad a=1, \ldots, n,
$$

in which $\mathbf{A}_{a}$ and $k_{a}$ are the normal (symmetric second-order tensor) to the hyper-plane and the reference stress (cohesion-like) for the $a^{\text {th }}$ plane, respectively. Such an approximation is presented in Figure 1 with four hyper-planes, two dashed and two solid lines, the latter two labeled $b$ and $c$. It is also convenient in what follows to introduce the new variables $s_{a}$ ("slack" variable) which define the distance between the stress point and the boundary of the linearized strength domain:

$$
\mathbf{A}_{a}: \boldsymbol{\sigma}-k_{a}+s_{a}=0 \quad \text { with } \quad s_{a} \geq 0 .
$$

The same graphical method proposed above is used to construct the support function of the linearized strength domain, referred to as $G_{L}$. The translation of the hyper plane of normal $\hat{\mathbf{d}}$ towards $G_{L}$ leads to the contact at the corner denoted $\boldsymbol{\sigma}^{L *}$. It corresponds to the intersection of two hyper-planes of normal $\mathbf{A}_{b}$ and $\mathbf{A}_{c}$ in our specific illustration. The virtual rate of deformation tensor has to be oriented within the cone defined by these two normals. Consequently and more generally, the virtual rate of deformation is linearly related to the normals of the various hyper-planes defining the contact point

$$
\mathbf{d}(\hat{\mathbf{U}})=\sum_{a=1}^{n} \mathbf{A}_{a} \hat{\lambda}_{a} \quad \text { with } \quad \hat{\lambda}_{a} \geq 0
$$


where $\hat{\lambda}_{a}$ are the non-negative virtual deformation components. In the example of Figure 1, the $\hat{\lambda}_{a}$ associated to the dashed lines are zero and the only strictly positive scalars are related to the planes $b$ and $c$. Furthermore, the support function of the linearized criterion has the following properties

$$
\pi_{L}(\hat{\mathbf{d}})=\sum_{a=1}^{n} \mathbf{A}_{a} \hat{\lambda}_{a}: \boldsymbol{\sigma}^{L *}=\sum_{a=1}^{n} \hat{\lambda}_{a} k_{a} \geq \pi(\hat{\mathbf{d}}),
$$

the second equality being a consequence of $\boldsymbol{\sigma}^{L *}$ belonging to each activated hyper-plane (non zero $\hat{\lambda}_{a}$ ) and on account of (8), which is then an equality.

The concept of support function is now used to derive the upper bound to the loading scalar $\alpha$. The internal work defined in (2) is bounded by above with

$$
\mathcal{P}_{\text {int }}(\hat{\mathbf{U}}) \leq \int_{\Omega} \pi_{L}(\hat{\mathbf{d}}) d V,
$$

so that the theorem of virtual power provides

$$
\alpha \int_{\partial \Omega_{T}} \mathbf{T}^{o} \cdot \hat{\mathbf{U}} d S \leq \int_{\Omega} \pi_{L}(\hat{\mathbf{d}}) d V-\int_{\Omega} \rho \mathbf{g} \cdot \hat{\mathbf{U}} d V, \quad \forall \hat{\mathbf{U}} \quad K A .
$$

The right-hand side provides the upper bound $\alpha_{U}$, after proper normalization in the left-hand side. The upper bound theorem, referred here as the maximum strength theorem, is thus summarized as the minimization problem with respect to the velocity fields

$$
\begin{array}{ll}
\text { minimize } & \alpha_{U}=\int_{\Omega}\left\{\sum_{a=1}^{n} \hat{\lambda}_{a} k_{a}-\rho \mathbf{g} \cdot \hat{\mathbf{U}}\right\} d V \\
\text { subject to } & \mathbf{d}(\hat{\mathbf{U}})=\sum_{a=1}^{n} \mathbf{A}_{a} \hat{\lambda}_{a} \quad \forall \mathbf{x} \in \Omega, \\
& \int_{\partial \Omega_{T}} \mathbf{T}^{o} \cdot \hat{\mathbf{U}} d S=1, \\
& \hat{\lambda}_{a} \geq 0 \quad \forall \mathbf{x} \in \Omega, \\
& \hat{\mathbf{U}} \in \mathcal{S}_{u}=\left\{\hat{\mathbf{U}} \mid \hat{\mathbf{U}}=0 \quad \forall \mathbf{x} \in \partial \Omega_{u}\right\} .
\end{array}
$$

\subsection{Spatial discretization and interpolation of the velocity field}

The spatial discretization and the interpolation of the velocity field as well as of the virtual deformation components $\hat{\lambda}_{a}$ are now introduced.

The domain of interest $\Omega$ is approximated by the domain $\Omega^{h}$ where the boundary corresponds to a series of straight segments or planar surfaces, as illustrated for the 2D case in Figure $2 \mathrm{a}$. The rest of this section presents the $2 \mathrm{D}$ element, the generalization to $3 \mathrm{D}$ is postponed to Appendix B. The interior of $\Omega^{h}$ is partitioned in $q$ six-noded triangles ( $q=11$ in Figure $2 \mathrm{~b}$ ). Note that the mid-side nodes are at the same distance from the two nodes at the adjacent vertices. The virtual velocities within a six-noded triangle are interpolated in terms of the nodal virtual velocities.

The velocity interpolation over a $2 \mathrm{D}$ element is

$$
\hat{\mathbf{U}}^{h}=\sum_{i=1}^{3} \zeta_{i}\left(2 \zeta_{i}-1\right) \hat{\mathbf{U}}_{i}+4\left[\zeta_{1} \zeta_{2} \hat{\mathbf{U}}_{4}+\zeta_{3} \zeta_{2} \hat{\mathbf{U}}_{5}+\zeta_{1} \zeta_{3} \hat{\mathbf{U}}_{6}\right],
$$


a)

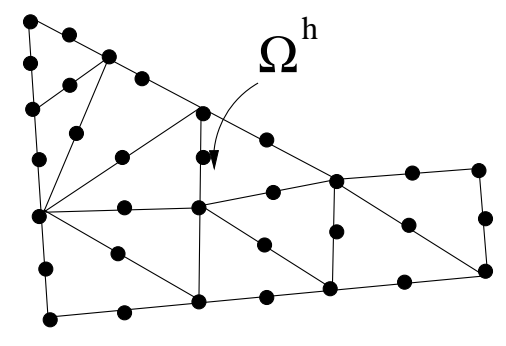

b)

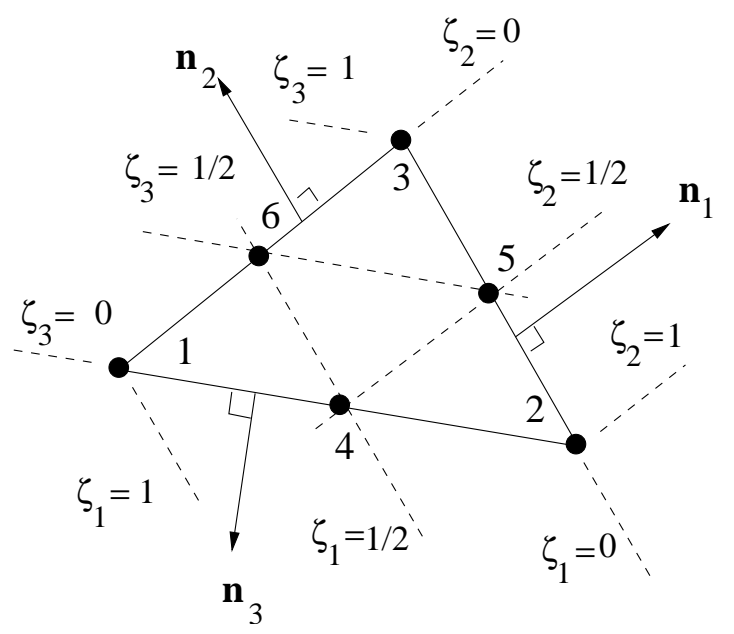

Figure 2: Discretization of the domain $\Omega$ by six-noded triangles, a). The six-noded triangle local node numbering and the definition of the area-coordinates, $b$ )

in terms of the area-coordinate $\zeta_{i}$ defined in Figure $2 \mathrm{~b}$ and the six nodal velocities $\hat{\mathbf{U}}_{i}$. The interpolated fields, denoted with the letter $h$ in upper-script, define a set of kinematically admissible fields

$$
\mathcal{S}_{u}^{h}=\left\{\hat{\mathbf{U}}^{h} \mid \hat{\mathbf{U}}_{i}=0 \text { if node } i \text { on } \quad \partial \Omega_{u}^{h}\right\} \subset \mathcal{S}_{u},
$$

which is a subset of $\mathcal{S}_{u}$. Consider that there are $m$ degrees of freedom set to zero by the above boundary condition and define the equivalent, global linear system

$$
[H]\{\hat{U}\}=\{0\}_{m},
$$

in which $[H]$ is the $m \times p$ matrix with components set either to zero or one. The column vector $\{\hat{U}\}$ in (17) is the global vector of nodal velocities which has, say, $p$ components (twice the total number of nodes in 2D). The notation in the left-hand side of (17) implies a matrix multiplication resulting in a column vector of length $m$. Note also in the right hand-side of (17) that the subscript $m$ defines the vector length, again for sake of clarity.

The element velocity interpolation is conveniently written in matrix notation as

$$
\left\{\hat{U}^{h}\right\}=\left[N_{u}\right]^{e}\{\hat{U}\}^{e},
$$

in which $\{\hat{U}\}^{e}$ is the column vector containing the local nodal velocities (length of 12) and $\left[N_{u}\right]^{e}$ is the $2 \times 12$ matrix of shape functions based on (15). The notation in (18) and in what follows for local array includes the letter $e$ in superscript to avoid any confusion with the global arrays. To compute the virtual rate of deformation, we first consider the gradient of the area-coordinate which are the constant vectors $\nabla \zeta_{i}=-\mathbf{n}_{i} l_{i} / A$ and are oriented opposite to the unit, external normal to the side opposite to node $i$, Figure $2 \mathrm{~b}$. Their norms are set by the length $l_{i}$ of the side $i$ divided by the area $A$ of the element. The gradient to the interpolated velocity (15) is thus

$$
\begin{aligned}
\nabla \hat{\mathbf{U}}^{h}= & -\sum_{i=1}^{3} \frac{l_{i}}{A}\left(4 \zeta_{i}-1\right) \hat{\mathbf{U}}_{i} \otimes \mathbf{n}_{i} \\
& -\frac{4}{A}\left[\hat{\mathbf{U}}_{4} \otimes\left(\zeta_{1} l_{2} \mathbf{n}_{2}+\zeta_{2} l_{1} \mathbf{n}_{1}\right)+\hat{\mathbf{U}}_{5} \otimes\left(\zeta_{2} l_{3} \mathbf{n}_{3}+\zeta_{3} l_{2} \mathbf{n}_{2}\right)+\hat{\mathbf{U}}_{6} \otimes\left(\zeta_{1} l_{3} \mathbf{n}_{3}+\zeta_{3} l_{1} \mathbf{n}_{1}\right)\right]
\end{aligned}
$$


which is proportional to the area-coordinates (note that $\sum_{i}^{3} \zeta_{i}=1$ ). This gradient is now used to construct the virtual rate of deformation tensor $\hat{\mathbf{d}}^{h}$ which is represented by the column vector $\left\{\hat{d}^{h}\right\}^{e}={ }^{t}\left(\hat{d}_{11}^{h}, \hat{d}_{22}^{h}, \hat{2} d_{12}^{h}\right)^{e}$ (note that a line vector is limited by parentheses and that the transpose of a column vector, denoted by a upperscript $t$ to the left, is a line vector). The virtual rate of deformation vector is then expressed locally in terms of the nodal velocities

$$
\left\{\hat{d}^{h}\right\}=[B]_{3 \times 12}^{e}\{\hat{U}\}^{e},
$$

in which the local $[B]^{e}$ operator is the $3 \times 12$ matrix for each element constructed from (19).

Attention is now turned to the local interpolation of the virtual deformation components $\hat{\lambda}_{a}$ defined in (10). It is proposed that these $n$ scalars be interpolated linearly

$$
\left\{\hat{\lambda}^{h}\right\}_{n}^{e}=\left[N_{\lambda}\right]^{e}\{\hat{\lambda}\}_{3 n}^{e}
$$

in terms of the vector of nodal values containing the $\hat{\lambda}_{a}$ 's at the three vertices. The shape function $N_{\lambda}$ of node $i$ is thus simply the area coordinate $\zeta_{i}$. This choice of interpolation implies that there is no continuity across the elements and the vector $\{\hat{\lambda}\}^{e}$ (3n components) is indeed specific to each element. The local interpolation of the $\hat{\lambda}_{a}$ 's is linear in the area-coordinates, as the interpolation of the virtual rate of deformation in (19) and (20). Consequently, the equality between the virtual rate of deformation and the linear combination of the stress normals in (10) is satisfied point-wise over each element by enforcing it at three specific points. The nodes at the vertices of each element are chosen for that purpose. The resulting system of equations reads

$$
[\mathcal{B}]^{e}\{\hat{U}\}^{e}=[\mathcal{A}]_{9 \times 3 n}^{e}\{\hat{\lambda}\}^{e}
$$

in which the $9 \times 12[\mathcal{B}]^{e}$ and the $9 \times 3 n[\mathcal{A}]$ matrices are defined by

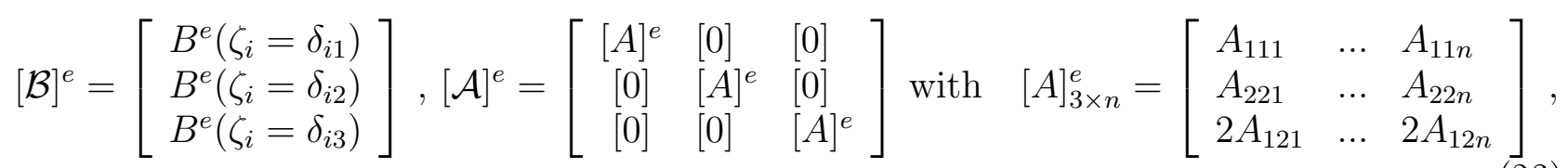

in which $\delta_{i j}$ is the Kronecker delta and the $A_{i j a}$ are the $i j$-component of the normal $\mathbf{A}_{a}$ to the $a^{\text {th }}$ hyper-plane.

To prepare the grounds for the discretization of the pre-defined discontinuities, it is convenient to multiply both sides of $(22)$ by $\frac{1}{3} A$, a third of the element area. In addition, the new scaled variables $\{\bar{\lambda}\}^{e} \equiv\left\{\frac{1}{3} A \hat{\lambda}\right\}^{e}$ are introduced so that (22) is replaced by

$$
[\overline{\mathcal{B}}]^{e}\{\hat{U}\}^{e}=[\mathcal{A}]^{e}\{\bar{\lambda}\}^{e} .
$$

The matrix $[\overline{\mathcal{B}}]^{e}=\frac{1}{3} A[\mathcal{B}]^{e}$ is represented in closed form as

$$
[\overline{\mathcal{B}}]_{9 \times 12}^{e}=-\frac{1}{6}\left[\begin{array}{cccccc}
3 \mathrm{P}_{1} & -\mathrm{P}_{2} & -\mathrm{P}_{3} & 4 \mathrm{P}_{2} & 0 & 4 \mathrm{P}_{3} \\
-\mathrm{P}_{1} & 3 \mathrm{P}_{2} & -\mathrm{P}_{3} & 4 \mathrm{P}_{1} & 4 \mathrm{P}_{3} & 0 \\
-\mathrm{P}_{1} & -\mathrm{P}_{2} & 3 \mathrm{P}_{3} & 0 & 4 \mathrm{P}_{2} & 4 \mathrm{P}_{1}
\end{array}\right]
$$

in terms of the $3 \times 2$ matrix

$$
\left[\mathrm{P}_{i}\right]=l_{i}\left[\begin{array}{cc}
n_{1}^{i} & 0 \\
0 & n_{2}^{i} \\
n_{2}^{i} & n_{1}^{i}
\end{array}\right],
$$

with $\left(n_{1}^{i}, n_{2}^{i}\right)$ being the two components of the unit outward normal to side $i$ (opposite node $i$ ). Equations (25) and (26) are derived from (19). It is noted that $[\overline{\mathcal{B}}]^{e}$ is well defined regardless 


\begin{tabular}{|l|l|}
\hline Symbol & definition \\
\hline \hline$q$ & number of elements \\
\hline$p$ & total number of velocity degrees of freedom \\
\hline$m$ & total number of velocity degrees of freedom set to zero (bound. cond.) \\
\hline$n$ & number of hyper-planes in stress space to bound the strength domain \\
\hline \hline
\end{tabular}

Table 1: Various definitions related to the discretization, the different interpolations and the linearization of the strength domains.

of the element area. This feature is of key importance in the incorporation of kinematically admissible velocity discontinuities as discussed at the end of this section.

In summary, the minimization problem (14), after discretization and interpolation, is written as

$$
\begin{array}{cl}
\text { minimize } & \alpha_{U}={ }^{t}\{k\}_{q 3 n}\{\bar{\lambda}\}-{ }^{t}\{G\}_{p}\{\hat{U}\} \\
\text { subject to } & {[\overline{\mathcal{B}}]_{9 q \times p}\{\hat{U}\}=[\mathcal{A}]_{9 q \times q 3 n}\{\bar{\lambda}\},} \\
& {[H]_{m \times p}\{\hat{U}\}=\{0\}_{m},} \\
& { }^{t}\left\{T_{0}\right\}\{\hat{U}\}=1, \\
& \{\bar{\lambda}\} \geq\{0\},
\end{array}
$$

in which the size of the global vectors and matrices could be estimated with the definitions summarized in Table 1. Note that a vectorial inequality should be interpreted as a series of inequalities for the corresponding components on the two sides. The $n$ successive components of the global $\{k\}$ vectors, for a given node in a given element, are the reference stresses $k_{a}$ defined in (9), assumed to be constant over each element for sake of simplicity. The vector $\{G\}$ requires a global assembly (several elements contributes to the same degree of freedom), the contribution of a single element being ${ }^{t}\{G\}^{e}=\rho A\left(0 ; 0 ; 0 ; 0 ; 0 ; 0 ; g_{x} / 3 ; g_{y} / 3 ; g_{x} / 3 ; g_{y} / 3 ; g_{x} / 3 ; g_{y} / 3\right)$, assuming a constant mass density and body force per element. The vector $\left\{T_{0}\right\}$ requires also a global assembly and its expression depends on the distribution of the applied force $\mathbf{T}_{0}$ on the boundary $\partial \Omega_{T}$.

\subsection{The dual problem}

The objective is now to construct the dual problem to (27) following the classical argument known in Linear Programming and presented for sake of completeness in Appendix A. To comply with the structure of the primal problem presented there, decompose the vector of nodal velocities $\{\hat{U}\}$ into two vectors of unknowns $\left\{\hat{U}^{+}\right\}$and $\left\{\hat{U}^{-}\right\}$with the conditions:

$$
\{\hat{U}\}=\left\{\hat{U}^{+}\right\}-\left\{\hat{U}^{-}\right\} \quad \text { with } \quad\left\{\hat{U}^{+}\right\} \geq\{0\} \quad \text { and } \quad\left\{\hat{U}^{-}\right\} \geq\{0\} .
$$


The primal problem (27) then becomes

$$
\begin{array}{ll}
\text { minimize } & \alpha_{U}=\left({ }^{t}\{k\} ;-{ }^{t}\{G\} ;{ }^{t}\{G\}\right)\left\{\begin{array}{c}
\{\bar{\lambda}\} \\
\left\{\hat{U}^{+}\right\} \\
\left\{\hat{U}^{-}\right\}
\end{array}\right\}, \\
\text {subject to } & {\left[\begin{array}{ccc}
{[\mathcal{A}]} & -[\overline{\mathcal{B}}] & {[\overline{\mathcal{B}}]} \\
{[0]} & {[H]} & -[H] \\
(0) & { }^{t}\left\{T_{0}\right\} & -{ }^{t}\left\{T_{0}\right\}
\end{array}\right]\left\{\begin{array}{c}
\{\bar{\lambda}\} \\
\left\{\hat{U}^{+}\right\} \\
\left\{\hat{U}^{-}\right\}
\end{array}\right\}=\left\{\begin{array}{c}
\{0\}_{9 q} \\
\{0\}_{m} \\
1
\end{array}\right\},} \\
& \left({ }^{t}\{\bar{\lambda}\} ;{ }^{t}\left\{\hat{U}^{+}\right\} ;{ }^{t}\left\{\hat{U}^{-}\right\}\right) \geq(0)_{3 q n+2 p} .
\end{array}
$$

The dual problem, following the results presented in Appendix A reads

$$
\begin{aligned}
& \operatorname{maximize} \quad \alpha_{U}=\left((0)_{9 q} ;(0)_{m} ; 1\right)\left\{\begin{array}{l}
\{\tilde{\sigma}\} \\
\{\tilde{R}\} \\
\alpha_{U}
\end{array}\right\} \\
& \text { subject to } \quad\left[\begin{array}{rrr}
{ }^{t}[\mathcal{A}] & {[0]} & \{0\} \\
-{ }^{t}[\overline{\mathcal{B}}] & { }^{t}[H] & \left\{T_{0}\right\} \\
{ }^{t}[\overline{\mathcal{B}}] & -{ }^{t}[H] & -\left\{T_{0}\right\}
\end{array}\right]\left\{\begin{array}{l}
\{\tilde{\sigma}\} \\
\{\tilde{R}\} \\
\alpha_{U}
\end{array}\right\}+\left\{\begin{array}{l}
\left\{\tilde{s}_{\lambda}\right\} \\
\left\{\tilde{s}^{+}\right\} \\
\left\{\tilde{s}^{-}\right\}
\end{array}\right\}=\left\{\begin{array}{c}
\{k\} \\
-\{G\} \\
\{G\}
\end{array}\right\} \text {, } \\
& \left({ }^{t}\left\{\tilde{s}_{\lambda}\right\} ;{ }^{t}\left\{\tilde{s}^{+}\right\} ;{ }^{t}\left\{\tilde{s}^{-}\right\}\right) \geq(0)_{3 n q+2 p},
\end{aligned}
$$

in which $3 n q+2 p$ slack variables have been introduced. Those variables are eliminated to provide the equivalent optimization problem

$$
\begin{array}{ll}
\text { maximize } & \alpha_{U} \\
\text { subject to } & { }^{t}[\overline{\mathcal{B}}]\{\tilde{\sigma}\}={ }^{t}[H]\{\tilde{R}\}+\alpha_{U}\left\{T_{0}\right\}+\{G\}, \\
& { }^{t}[\mathcal{A}]\{\tilde{\sigma}\} \leq\{k\} .
\end{array}
$$

A physical interpretation of the dual variables is now tentatively proposed. The $\tilde{\sigma}$ (3 components at each vertex) can be seen as stress-like quantities from the dimension point of view. The $\tilde{R}$ (1 component for each constrained velocity degree of freedom) are like reaction forces. The set of equalities in (31) is then seen as an expression of the balance of the internal and external forces for the dual problem. It is for that reason that the matrix $[\overline{\mathcal{B}}]$ is often referred to as the pseudo-equilibrium matrix. This interpretation is however limited in the sense that none of the above stress-like quantities are derived from a statically admissible stress field. We can only state that the dualization has provided a max-problem which is convenient to search for the upper bound to the tectonic force, as it is shown next.

The set of inequalities in (31) is due to the linearized strength domains introduced in (8). The linearization of the strength domain tends to the original non-linear domain in the limit of an infinite number of hyper-planes. In this limit, the linearized strength domain can be 
replaced by the original non-linear domain so that (31) becomes

$$
\begin{array}{ll}
\text { maximize } & \alpha_{U} \\
\text { subject to } & { }^{t}[\overline{\mathcal{B}}]\{\tilde{\sigma}\}={ }^{t}[H]\{\tilde{R}\}+\alpha_{U}\left\{T_{0}\right\}+\{G\}, \\
& G\left(\tilde{\sigma}_{i}\right) \leq 0 \text { for } i=1 \text { to } 3, \text { for each element in } \Omega^{h} .
\end{array}
$$

It is this problem which is set up with SARPP (2008) and solved with MOSEK (2008) in 2D and 3D. The details of the 3D formulation are presented in Appendix B. Appendix C establishes the link with the conic programming algorithms adopted in MOSEK (2008). It is emphasized that although the final problem is reminiscent of a classical lower bound construction, it does in fact result in a rigorous upper bound. The procedure of $(i)$ linearizing the strength domain, (ii) setting up a discrete upper bound problem, (iii) constructing the dual problem, and finally (iv) replacing the linearized strength domain by the original non-linear domain provides a completely general approach to numerical upper bound limit analysis. In contrast, numerical formulations based on the primal form of the upper bound theorem are highly dependent on the particular expression of the support function.

\subsection{Velocity discontinuities}

The ability to incorporate kinematically admissible velocity discontinuities across surfaces of known geometry is often desired as for example in the $2 \mathrm{D}$ and $3 \mathrm{D}$ wedge problems considered next and in the companion paper. The internal work for the continuum problem in (2) should then be amended to account for the virtual power $\mathbf{T} \cdot \llbracket \hat{\mathbf{U}} \rrbracket$ in which $\mathbf{T}$ is the stress vector dual to the jump in the virtual velocities. These surfaces have specific material properties reflected by a strength domain which would be represented in a stress space of reduced dimension. Typically this dimension is two, corresponding to the resolved shear stress and the normal stress. The linearization of these strength domain, if necessary, would lead to additional nonnegative virtual deformation components $\hat{\lambda}_{J}$ introduced to decompose the velocity jump, in the same way the rate of deformation was presented in (10). These extra variables would be included into the upper bound problem (14). The dualization of Section 2.3 would have been done along the same line of thoughts with additional dual variables corresponding to stress-like vectors. Alternatively, we consider that a material discontinuity is simply an infinitely thin layer of material, likely of specific properties, but which is discretized similarly to the bulk region. The feasibility of this approach - and indeed, its equivalence to traditional kinematic formulation such as that of Sloan and Kleeman (1995) - was first demonstrated by Krabbenhoft et al. (2005) in the context of elements with a linear variation in the velocities.

In the present paper, elements with a quadratic variation of the velocities are used. The construction of the discontinuity with a zero-thickness patch of elements is possible thanks to the appropriate scaling with the element area, leading to the introduction of the matrix $[\overline{\mathcal{B}}]^{e}$ in (25). This matrix is well defined even for an element area identically set to zero. Therefore, as a direct extension of the linear velocity element, we propose to construct discontinuities of known position and geometry as patches of two zero-thickness quadratic elements, as illustrated in Figure 3. The resulting velocity jumps are quadratic and should have relatively moderate influence on the accuracy of the limit load. It is of interest to reduce the number of variables associated with a given discontinuity. For this purpose, the internal discontinuity velocities (which are not attached to either of the jointed regions) are expressed in terms of the others (the nodes attached to either side) in the following way: 


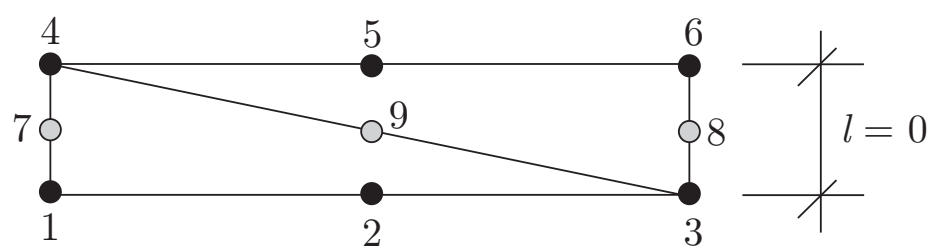

Figure 3: Kinematically admissible velocity discontinuity comprised of two quadratic elements of thickness $l$ set to zero.

$$
\hat{\mathrm{U}}_{7}=\frac{1}{2}\left(\hat{\mathrm{U}}_{1}+\hat{\mathrm{U}}_{4}\right), \quad \hat{\mathrm{U}}_{8}=\frac{1}{2}\left(\hat{\mathrm{U}}_{3}+\hat{\mathrm{U}}_{6}\right), \quad \hat{\mathrm{U}}_{9}=\frac{1}{2}\left(\hat{\mathrm{U}}_{2}+\hat{\mathrm{U}}_{5}\right)
$$

Other choices are of course possible, but numerical tests suggest that the above proposition is efficient, with only a marginal decrease in accuracy as compared to the case of a full quadratic discontinuity with three independent internal nodes Pastor (2006). It should be emphasized that this condensation restricts the velocities to vary linearly across a discontinuity. Along the discontinuity, the velocity jump is still quadratic in the tangential direction, in contrast to more traditional kinematic formulations (e.g. Pastor et al., 2008).

\section{Application to the 2D stability of accretionary wedges}

The objective of this section is to validate the numerical development with the example of the 2D stability of accretionary wedges, and in particular of cohesionless triangular wedges. There is an analytical solution (Dahlen, 1984) for that particular case which can also be obtained with the Mohr construction (Lehner, 1986).

The 3D wedge studied in the companion paper is presented in Figure 4a where the observer is seen exerting the force $Q$ on the back wall. His horizon is set on the décollement, the lower surface on which the wedge is resting, so that gravity is acting at the angle $\beta$ from the vertical direction. Of interest to this contribution is the wedge in the $2 \mathrm{D}$ central cross section ABC which angle is $\alpha+\beta$, Figure $4 \mathrm{~b}$. Geometrical and material parameters are provided in Table 2.

\subsection{The critical wedge theory}

\begin{tabular}{|l|l|l|l|}
\hline Symbol & definition & value & unit \\
\hline \hline$\alpha$ & topographic slope angle & variable & $\mathrm{deg}$ \\
\hline$\beta$ & décollement angle & 3 & $\mathrm{deg}$ \\
\hline$D$ & total length of the décollement & 50 & $\mathrm{~km}$ \\
\hline$\delta$ & thichness of décollement and back wall & $10^{-6} \mathrm{D}$ & $\mathrm{km}$ \\
\hline$\phi_{B W}$ & friction angle of the back wall & variable & $\mathrm{deg}$ \\
\hline$C_{B W}$ & cohesion of the back wall & 0. & $\mathrm{~Pa}$ \\
\hline$\phi_{D}$ & friction angle of the décollement & 15 & $\mathrm{deg}$ \\
\hline$C_{D}$ & cohesion of the décollement & 0. & $\mathrm{~Pa}$ \\
\hline$\phi_{b}$ & friction angle of the bulk material & 30 & $\mathrm{deg}$ \\
\hline$C_{b}$ & cohesion of the bulk material & 0. & $\mathrm{~Pa}$ \\
\hline$\rho$ & material density & 2200. & $\mathrm{~kg} / \mathrm{m}^{3}$ \\
\hline$g$ & gravity acceleration & 9.81 & $\mathrm{~m} / \mathrm{s}^{2}$ \\
\hline
\end{tabular}

Table 2: Geometrical and material parameters for the 2D applications unless they vary from one simulation to the other. The critical slope $\alpha_{c}$ is $3.38^{\circ}$ for this data set. 
a)

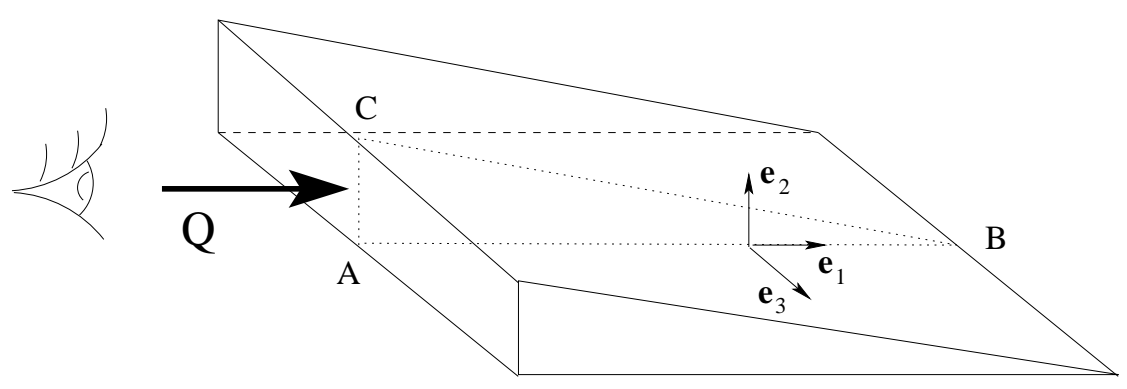

b)

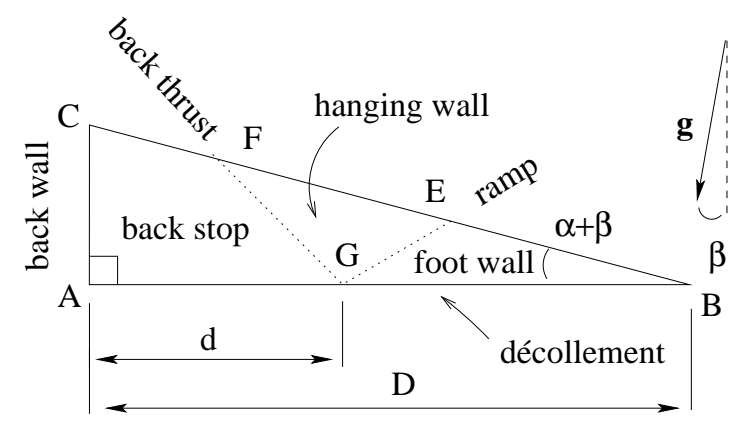

Figure 4: The 3D geometry of the accretionary wedge a) and the central cross-section considered for the 2D analysis, b).

The theory of the critical, cohesionless wedge is summarized as follows considering the slope of the décollement $\beta$ constant. For topographic angles $\alpha$ less than $\alpha_{c}$, the deformation occurs at the back of the structure which is said to be sub-critical. The failure mode is typically composed of a ramp and a back thrust, as illustrated in Figure 4b for 2D problems by the segments GE and GF, respectively. They correspond ideally to velocity discontinuities (Cubas et al., 2008). Material in the back stop is displaced parallel to the décollement before taking a trajectory parallel to the ramp at the crossing of the back thrust. The material in the hanging wall is over thrusting the material in the foot wall which is at rest. For sub-critical slope conditions $\left(\alpha<\alpha_{c}\right)$, the common root of the two discontinuities on the décollement is as much as possible to the back, so that point $\mathrm{F}$ is superposed on point $\mathrm{C}$. Only part of the décollement is activated, segment AG. For slope angles larger than $\alpha_{c}$, the deformation is to the front and the structure is said to be super-critical. In that instance, the failure mechanism (ramp, back thrust) collapses to a single point at the toe of the wedge, point $\mathrm{B}$, and the whole décollement is activated. The transition occurs exactly for $\alpha=\alpha_{c}$ and is marked by the potential activation of faulting everywhere within the wedge. The distance of the root of the failure mechanism, denoted $d$, is then undetermined. The analytical expression (Dahlen, 1984) for the critical slope angle is

$$
\alpha_{c}+\arcsin \left(\frac{\sin \alpha_{c}}{\sin \phi_{R}}\right)=-2 \beta+\arcsin \left(\frac{\sin \phi_{D}}{\sin \phi_{R}}\right)-\phi_{D}
$$

This interpretation of Dahlen's solution is in line with the results of Cubas and al. (2008) who applied the maximum strength theorem for the failure mechanism composed of a ramp and back thrust. Their minimization is in terms of three variables, the dips of the two velocity discontinuities and the position $d$ of their common root on the décollement. These analytical results pinpoint exactly the transition. The objective is now to repeat this analysis with the proposed numerical scheme without postulating the shape nor the position of the failure modes. 


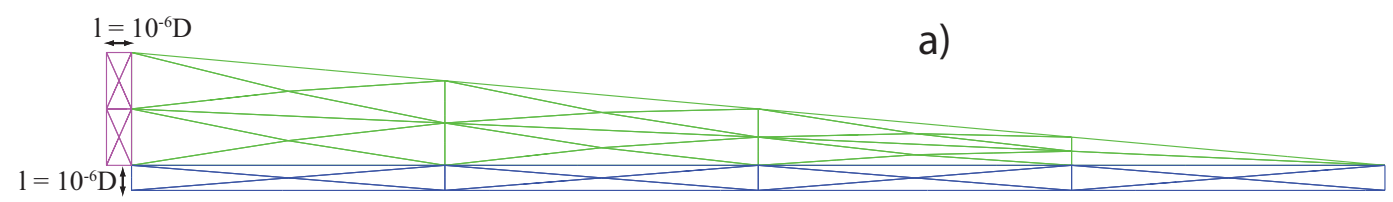

b) $\sigma_{\mathrm{e}}[\mathrm{MPa}]$

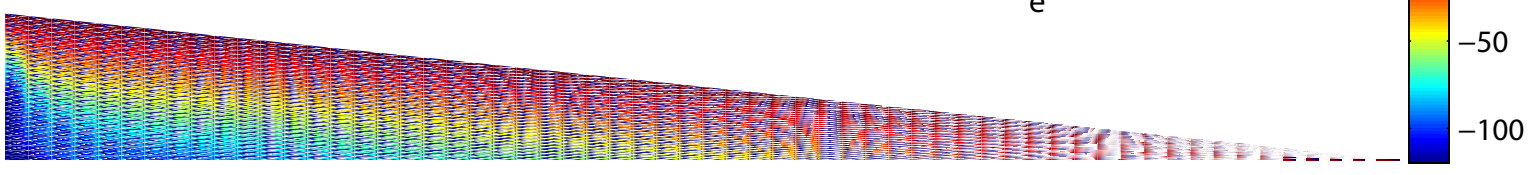

c) $\mathrm{P}[\mathrm{MPa}]$

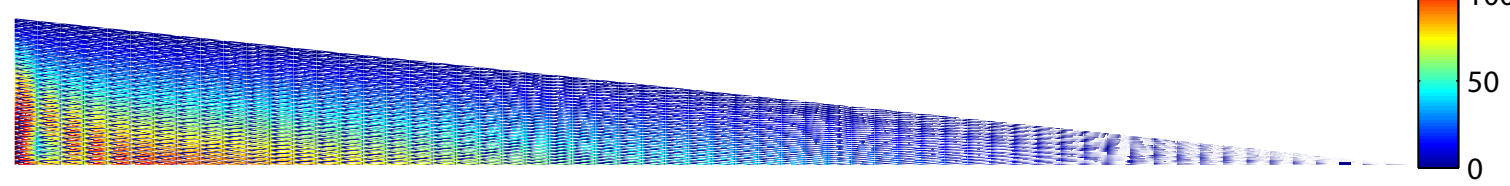

Figure 5: The type of mesh over the wedge, including the collapsed elements for the interfaces, a). The two invariants of the stress-like results, the in-plane mean stress $P \mathrm{~b}$ ), and the equivalent stress $\sigma_{e}$ for $\alpha=3^{\circ}$, c), corresponding to sub-critical slope conditions. Units: MPa.

The mesh considered for that problem consists of $60 \times 30$ cells, each composed of four crossed triangles, except for the cells at the toe composed of a single triangular element. A coarse mesh is presented in Figure 5a for sake of illustration. The collapsed cells for friction on the back wall and the décollement are also presented. The thickness of these two layers is set to $l=10^{-6} \mathrm{D}$ corresponding to the physical thickness of $5 \mathrm{~cm}$. There is a total of 7470 elements and 15123 nodes. The boundary conditions are as follows. The velocities on the lowest plane parallel to the décollement are prescribed to be zero (see equation 17). The horizontal component of the velocity at the rear of the back wall layer are set to one, leading to an algorithm slightly different from the general case presented in section 2 and discussed in Souloumiac (2008). The only material property not set in Table 2 is the back wall friction angle: $\phi_{B W}=30^{\circ}$.

The fundamental problem unknowns are the three stress components $\left(\sigma_{11}, \sigma_{22}\right.$ and $\left.\sigma_{12}\right)$ defined at the three vertices of each triangle, in the basis attached to the observer, Figure 4a. Results of the dual problem are presented in Figure 5b and $\mathrm{c}$ in terms of the equivalent shear stress and the in-plane mean stress defined in (4). These results are obtained for $\alpha=3^{\circ}$, corresponding to sub-critical conditions $\left(\alpha_{c}=3.38^{\circ}\right)$. It is tentatively proposed to interpret physically those stress fields although they are not statically admissible. The motivation for this proposition comes from the stress distribution which is mostly parallel to the topography. There is thus an invariance of the stress field with respect to the position along the free surface, the classical assumption in the critical wedge theory. This spatial dependence is altered close to the back wall for reasons which will be discussed in the next subsection. This variation occurs in a region of characteristic size less than the wedge height $H$ defined as $D \tan (\alpha+\beta)$.

The primal variables are the nodal velocities and they are also computed by the optimization code MOSEK (2008). They are used in Figure 6a to construct the boundary of the deformed mesh considering the virtual velocity as the actual velocity and taking a time step of arbitrary magnitude. The original domain boundaries correspond to the dashed lines. There is a forward motion of two triangular regions with boundaries delineated by dotted segments, which we 

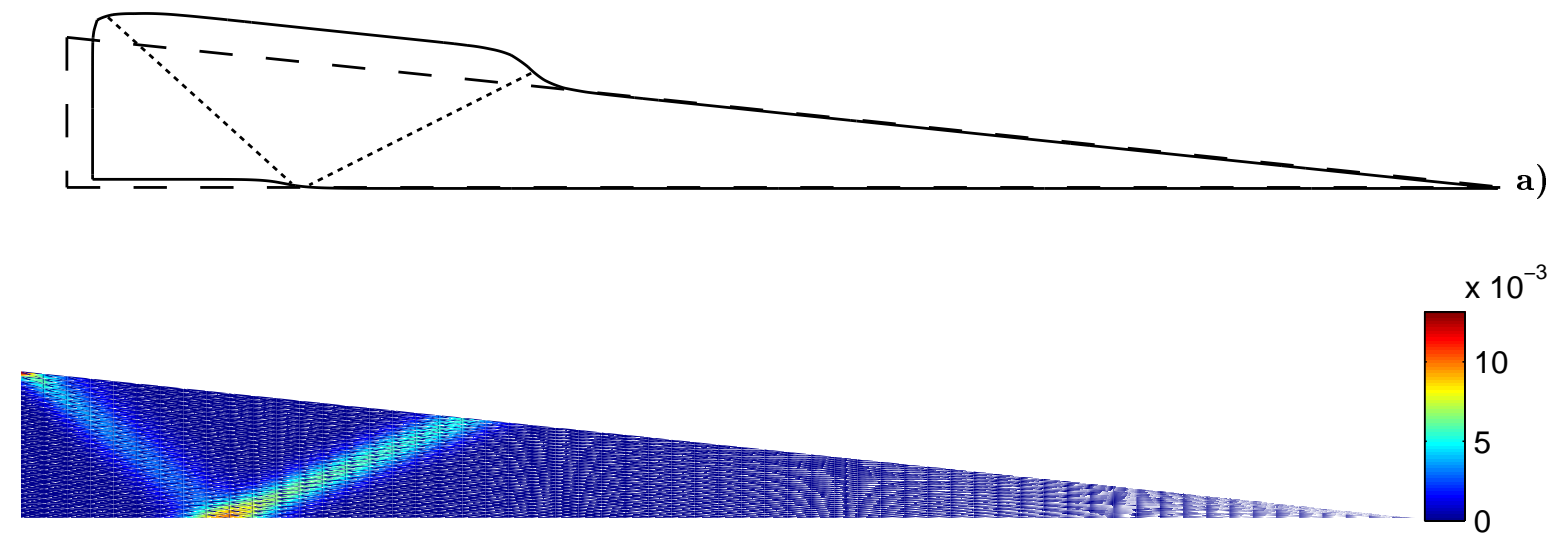

b)

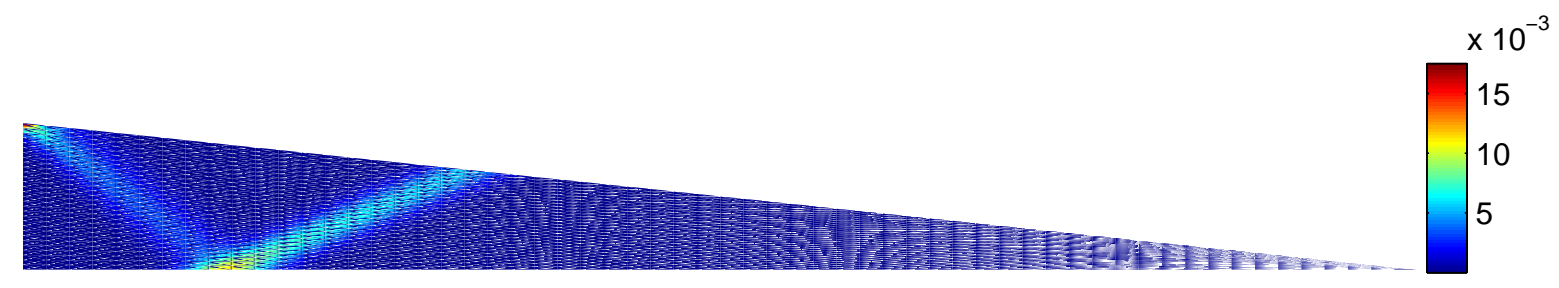

c)

Figure 6: Sub-critical slope conditions: $\alpha=3^{\circ}<\alpha_{c}$. The deformed boundary of the mesh based on the nodal velocities at the six nodes of each triangle, superposed to the original mesh in dashed lines, a). The dotted segments mark the core of the zone of localized virtual deformation and could be interpreted as the back thrust and the ramp. Iso-contours of the virtual volumetric $\hat{\theta}$ and the virtual equivalent shear strain $\hat{\gamma}$ are presented in b) and c), respectively.

propose to mark the ramp and the back thrust. The region most to the rear is the back stop and the other the hanging wall. Their boundaries are strips of localized deformation. The motion along the décollement ceases at the point where the ramp and the back are rooting. This interpretation of the failure mechanisms and more generally the analysis of the spatial gradient in the virtual rate of deformation tensor are facilitated with the introduction of the two invariants

$$
\hat{\theta}=\operatorname{tr}(\mathbf{d}(\hat{\mathbf{U}})), \quad \hat{\gamma}=\sqrt{\hat{\mathbf{d}}^{\prime}: \hat{\mathbf{d}}^{\prime}} \quad \text { with } \quad \hat{\mathbf{d}}^{\prime}=\hat{\mathbf{d}}-\frac{\hat{\theta}}{3} \boldsymbol{\delta}
$$

in which $\hat{\mathbf{d}}^{\prime}$ is the deviatory, virtual rate of deformation tensor. The first invariant is the virtual volumetric strain and the second, the virtual equivalent shear strain. They are plotted in $6 \mathrm{~b}$ and $\mathrm{c}$ over the original domain. One observes a strong virtual strain localization along the two directions at $23.5^{\circ}$ and $40.5^{\circ}$ corresponding to the expected dips of the ramp and back thrust. The virtual dilation $\hat{\theta}$ is of course more difficult to interpret physically, in the absence of any plasticity constitutive response. In particular, the vertical displacement along the décollement which marks its activation extent, and seen in Figure 6a, will not be interpreted beyond the constraint due to the structure of the support function in equation 7 . We know from Cubas and al. (2008) that the décollement is in condition (2) and the virtual velocity vector is at the angle $\phi_{D}$ from this surface, explaining the virtual opening necessary for the virtual sliding. This opening or dilation is necessary according to the definition of the support function. It is interesting to note that the dual problem leads to the same conclusion although the exact expression for the support fonction is not required.

Results for the topographic slope at criticality $\left(\alpha=\alpha_{c}\right)$ are presented in Figure 7 and consist of the deformed mesh and the distribution of the virtual equivalent shear strain $\hat{\gamma}$. Most of the 

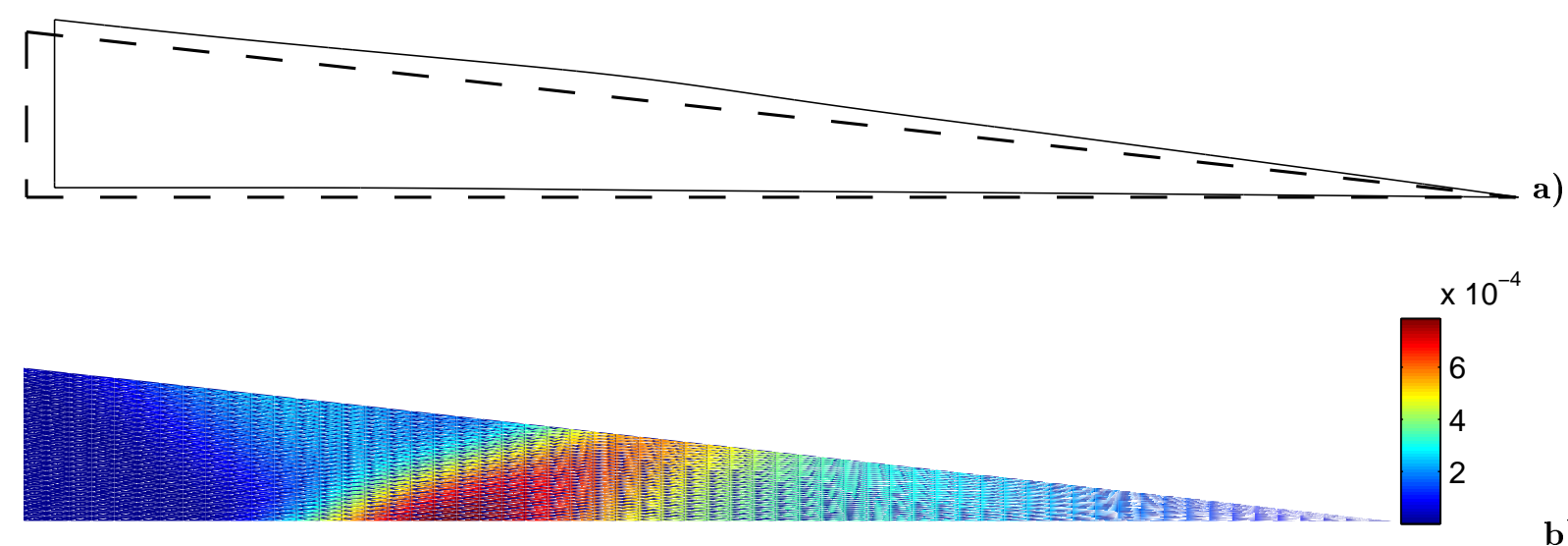

Figure 7: Critical slope conditions: $\alpha=\alpha_{c}$. The boundary of the deformed mesh superposed to the original mesh, dashed lines, a) and the iso-contours of equivalent-shear virtual-strain $\hat{\gamma}$ in b). The whole décollement is activated and the deformation is rather diffuse.

décollement appears to be activated and the virtual deformation in the bulk is mostly diffuse with a large ramp region which marks more the flexure of the domain than the tendency for the virtual strain to localize. Results for super-critical slope conditions are presented in Figure 8. The whole décollement is activated except at the toe where there are some mesh effects. The virtual deformation is zero in most of the wedge except in that specific region.
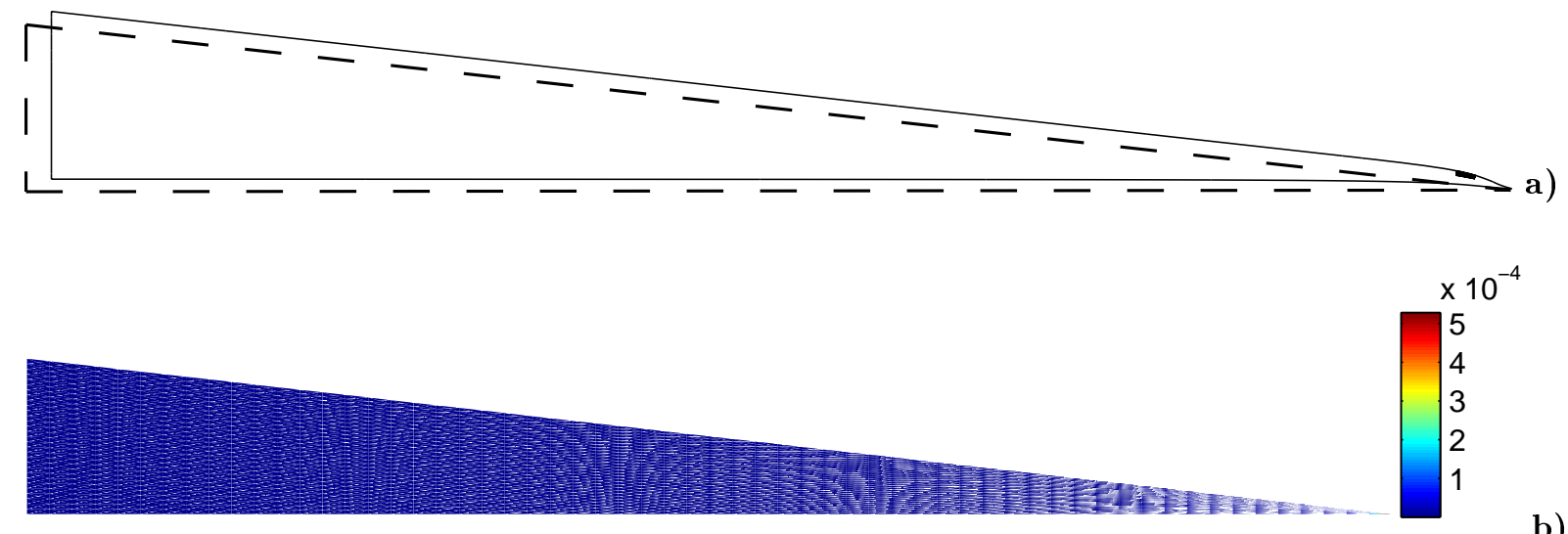

Figure 8: Super-critical slope conditions: $\alpha=3.5>\alpha_{c}$. The boundary of the deformed mesh superposed to the original mesh, dashed lines, a) and the iso-contours of equivalent-shear virtual-strain $\hat{\gamma}$ in b).

The results presented in Figures 6 to 8 illustrate that our numerical implementation does capture the stability of the perfectly-triangular wedge. The mode of failure is indeed with a ramp and back thrust system defined numerically as localized zone of virtual shear and dilation. Such strips are well described by sharp velocity discontinuities in analytical work (Cubas and al. 2008). The dips of the numerical failure system coincide with the analytical predictions.

This quantitative validation is continued by plotting in Figure 9 the distance $d$, positioning the root of the failure mechanism on the décollement, as a function of the topographic slope $\alpha$ (back wall friction $\phi_{B W}=30^{\circ}$ ). The dashed vertical line corresponds to Dahlen's critical slope of $\alpha_{c}=3.38^{\circ}$. The numerical results are presented as open circles linked by the series of solid segments. For $\alpha$ less than $\alpha_{c}, d$ is as small as possible to let the back thrust outcrop on the top surface. It is equal to the whole décollement length, $D$, for values larger then $\alpha_{c}$. 


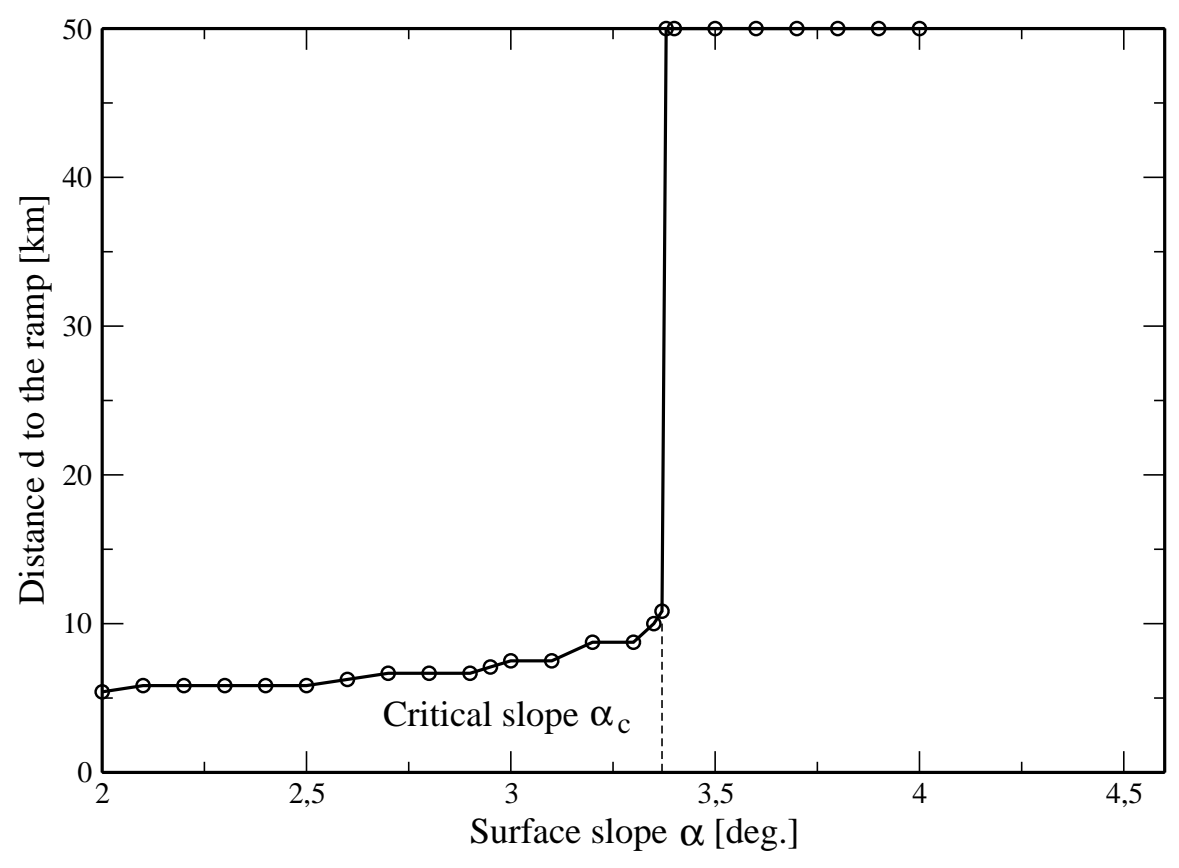

Figure 9: The position of the root of the ramp and back thrust on the décollement as a function of the topographic slope. The dashed line marks the analytical solution of Dahlen (1984). The back-wall friction angle is set to $\phi_{B W}=30^{\circ}$.

The numerical transition occurs exactly for the analytical value of the critical slope, further validating the numerical development.

\subsection{Influence of the friction on the back wall}

The series of $2 \mathrm{D}$ results are completed with a regard on the influence of the back wall friction. This analysis is certainly of most interest to the practitioners of numerical modeling and physicists reproducing with analogue materials in the laboratory the work of nature, where the concept of boundary conditions remains difficult to grasp (Schreurs et al., 2006). The topographic slope is set to $\alpha=3^{\circ}$, corresponding to sub-critical conditions so that failure should occur close to the back wall.

The upper bound in the tectonic force necessary to initiate failure is presented as a function of the back wall friction angle in Figure 10. The curve is approximately defined by two straight segments intersecting for the specific value $\phi_{B W}^{*}=5.8^{\circ}$. This critical value of the back wall friction angle marks also a change in the failure modes which are illustrated in Figure 11 with isocontours of virtual equivalent shear strain. For the smallest value $\phi_{B W}=3^{\circ}$, the failure mode is composed of a single ramp taking root on the décollement at the back wall contact. A triangular region is virtually moving up the ramp requiring shear along the back wall. For values of the friction angle close to $\phi_{B W}^{*}$, a fraction of the décollement is activated and the back thrust is intersecting the back wall at depth. The failure system favors the activation of the décollement to reduce sliding on the back wall. The transition to the straight ramp and back thrust takes place for $\phi_{B W}$ larger than $\phi_{B W}^{*}$, as illustrated in Figure 11d and e, for $7^{\circ}$ and $15^{\circ}$.

The dual stress field for the case of $\phi_{B W}=5.7^{\circ}$ is presented in Figure 12 in terms of the two invariants $\sigma_{e}$ and $P$, defined in (4). The main difference with the results obtained for $\phi_{B W}=30^{\circ}$ in Figure 5 is the absence of stress concentration at the bottom left corner of the wedge. The stress state seems to be only function of the distance to the topographic surface, 


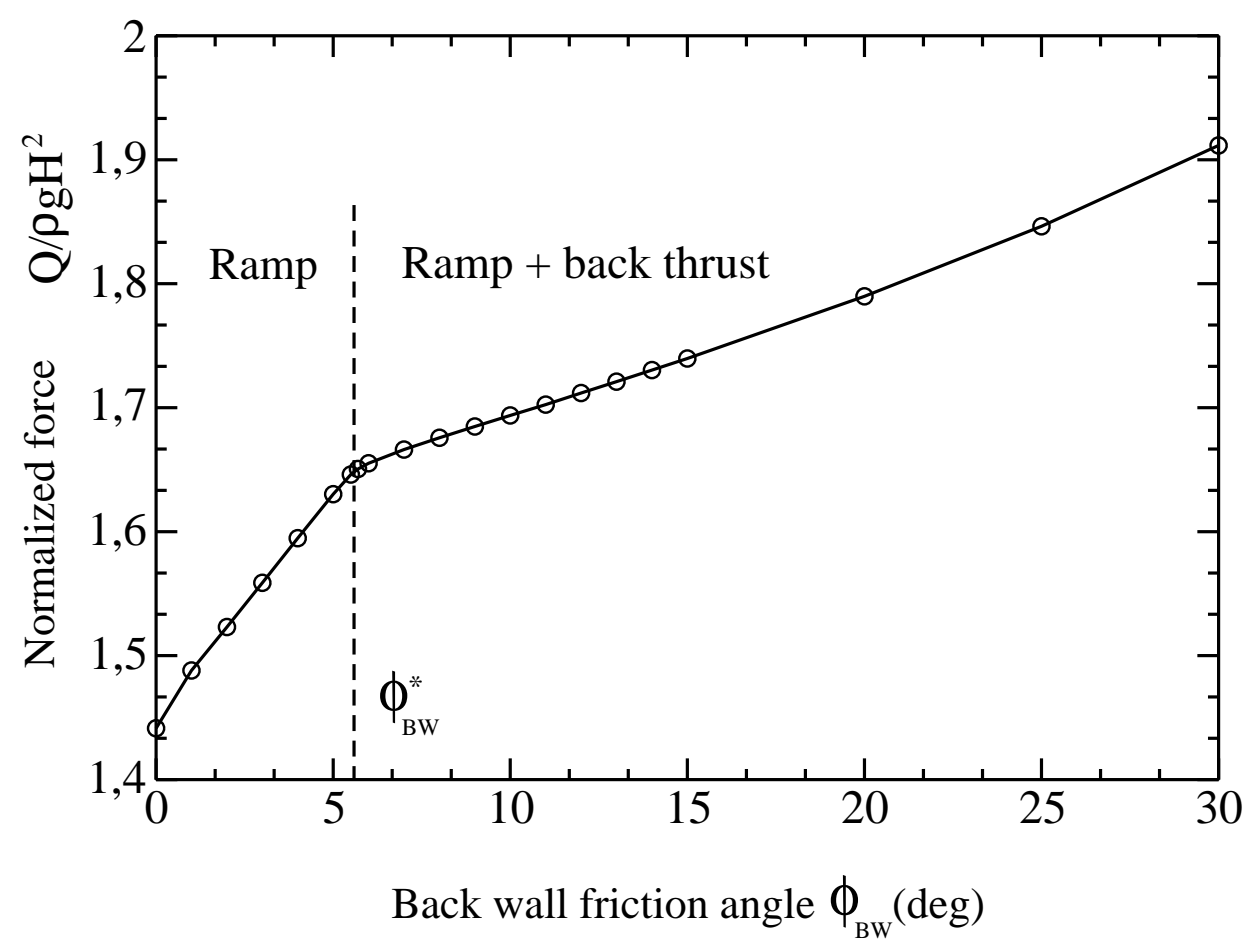

Figure 10: The normalized upper bound to the tectonic force as a function to the friction angles on the back wall.

a classical stress state in soil mechanics and used by Dahlen (1984) and Lehner (1986). This statically admissible stress state is used next to explain further the influence of the back wall friction on the failure mode.

The stress state is assumed to be independent of the position along the topography ( $\mathrm{x}$ coordinate in Figure 13a) and thus reads $\sigma_{x z}=\rho g z \sin \beta$ and $\sigma_{z z}=-\rho g z \cos \beta$. The stress vector $\boldsymbol{\sigma} \cdot \mathbf{n}$ acting on the facette no 1 in Figure 13a has the component $\left(\tau=-\sin \beta, \sigma_{n}=\right.$ $-\cos \beta$ ), once normalized by $\rho g z$, in the direct basis $(\mathbf{n}, \mathbf{t})$ shown in the same Figure. This stress vector corresponds to point $\mathrm{T}$ in the Mohr construction of Figure 13b where the normalized coordinates $\sigma_{n} / \rho g z$ and $-\tau / \rho g z$ are used to render consistent the stress sign convention and the use of the pole defined in what follows. The normalization in the absence of any cohesion for the Coulomb criterion renders the following construction applicable at any position within the wedge. The two Mohr circles which are presented are tangent to the Coulomb strength criterion. They represent the active and the passive stress state, respectively. We are interested by the passive circle defining the failure mode under compression at the back of the wedge. The pole $\mathrm{P}$ of this circle is defined by the remarkable property that any line oriented with the physical dip of the facette of interest (not its normal) and passing through $\mathrm{P}$ also intersects the Mohr circle at the point defining the relevant stress vector (see Mandl, 2005). This is clearly the case of facette 1 which was used to construct the pole. It is also true of the décollement dipping at $\beta$ which has the stress vector at point $R^{\prime}$. The zoom in the region of points $R^{\prime}$ and $T$ in Figure 13c shows that this point R' differs from point $\mathrm{R}$ which is the stress vector necessary to activate the décollement with friction angle $\phi_{D}$. The point $R^{\prime}$ is below $R$ and signals that the décollement friction is too large for this surface to be activated. Our choice of parameters does correspond to sub-critical conditions. The pole is further used to construct the stress vector of facette no 2, parallel to the back wall, which is at point $\mathrm{S}$ in the Mohr's construction. The value of the friction angle which would mark the activation of slip along the back wall is $\phi_{B W}^{*} \simeq 6^{\circ}$, 
within the accuracy of the measure with a protractor. This is certainly a sufficiently accurate approximation of the $5.7^{\circ}$ found numerically above.

If $\phi_{B W}$ is larger than $\phi_{B W}^{*}$, slip is prevented to occur on the back wall and the back stop can only glide on the décollement. For $\phi_{B W}$ smaller then $\phi_{B W}^{*}$, slip can occur on the back wall and the failure mechanism make use of that property to initiate the ramp at the back wall. The back stop is then part of the hanging wall. For the critical value $\phi_{B W}^{*}$, the numerical stress field coincides closely to the statically admissible field used in the Mohr's construction, see Figure 12. 


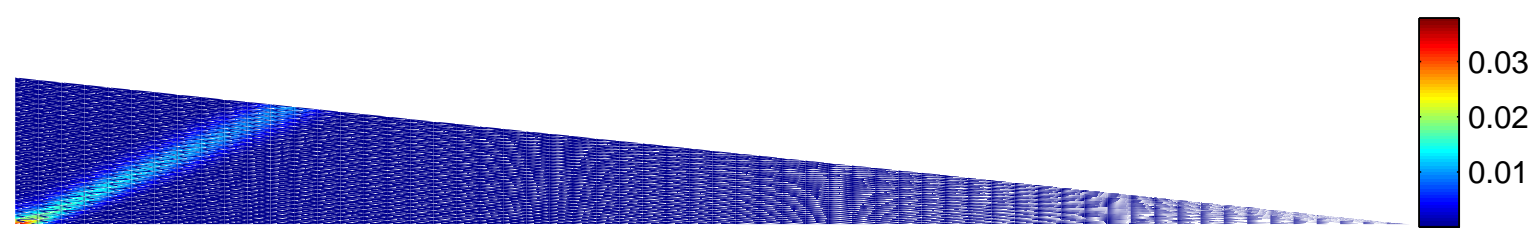

a)
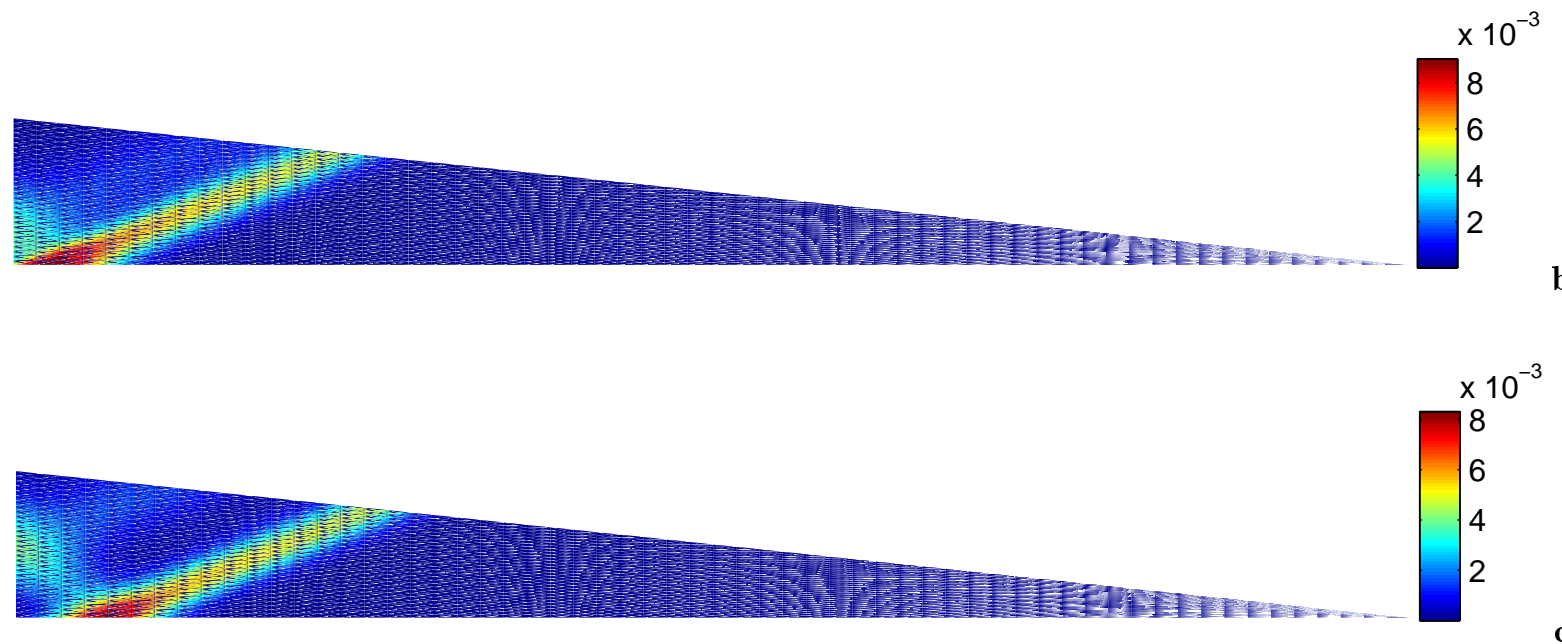

c)

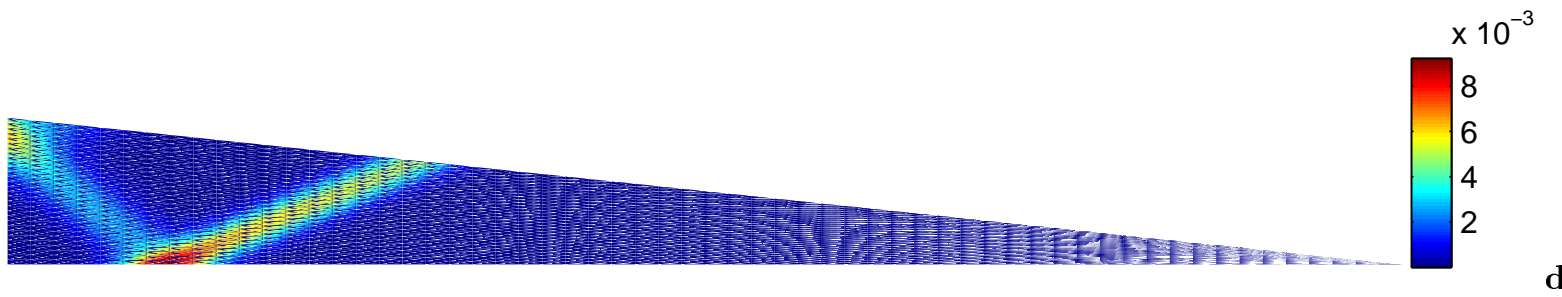

d)

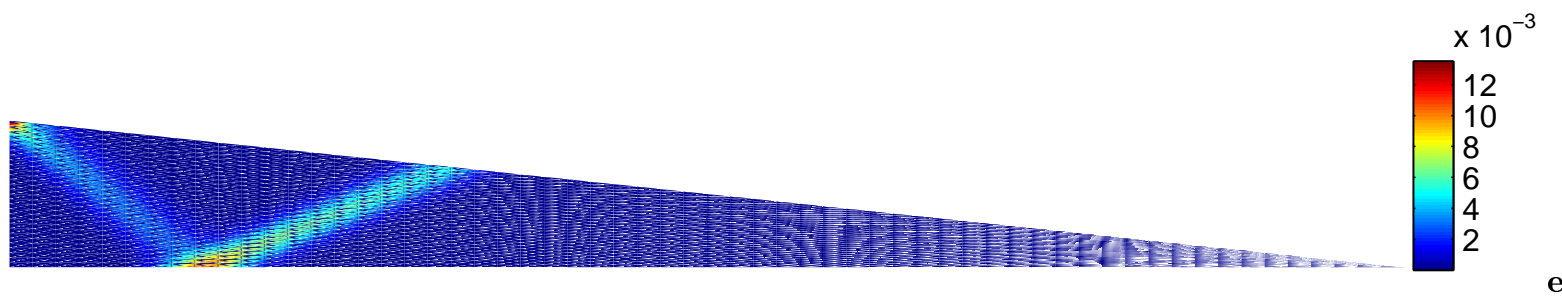

Figure 11: The failure mode for sub-critical topographic slope conditions $\left(\alpha=3^{\circ}<\alpha_{c}\right)$ for fives values of the back wall friction $\phi_{B W}$ set to $3^{\circ}, 5.5^{\circ}, 5.7^{\circ}, 7^{\circ}$ and $15^{\circ}$, in a) to e), respectively. Iso-contours of the virtual equivalent shear strain $\hat{\gamma}$. 
a) $\sigma_{e}[\mathrm{MPa}]$

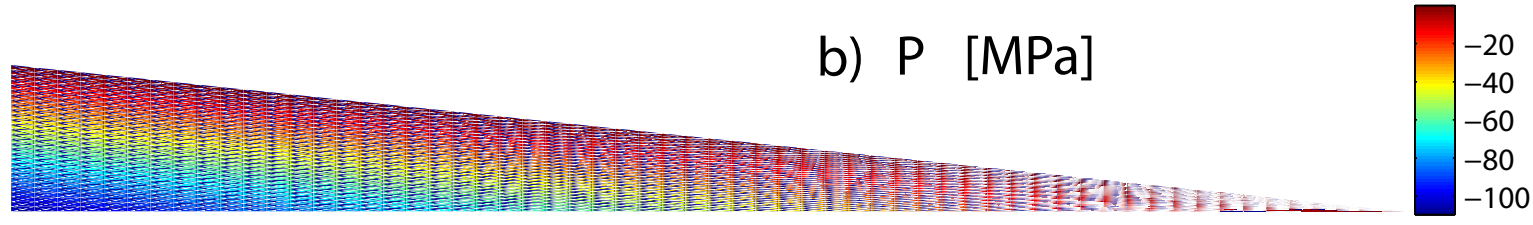

Figure 12: The two stress invariants, the equivalent shear $\sigma_{e}$ and the in-plane mean stress $P$ in a) and b), respectively, for a back wall friction $\phi_{B W}=5.7^{\circ}$ 
a)

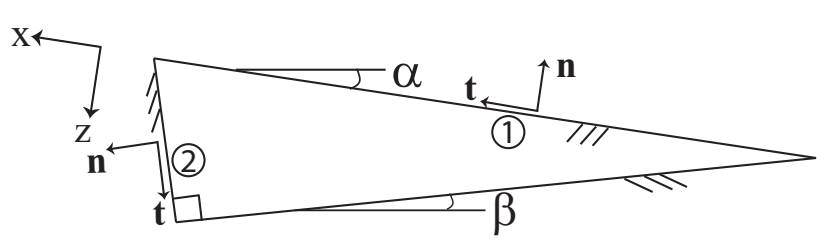

b)

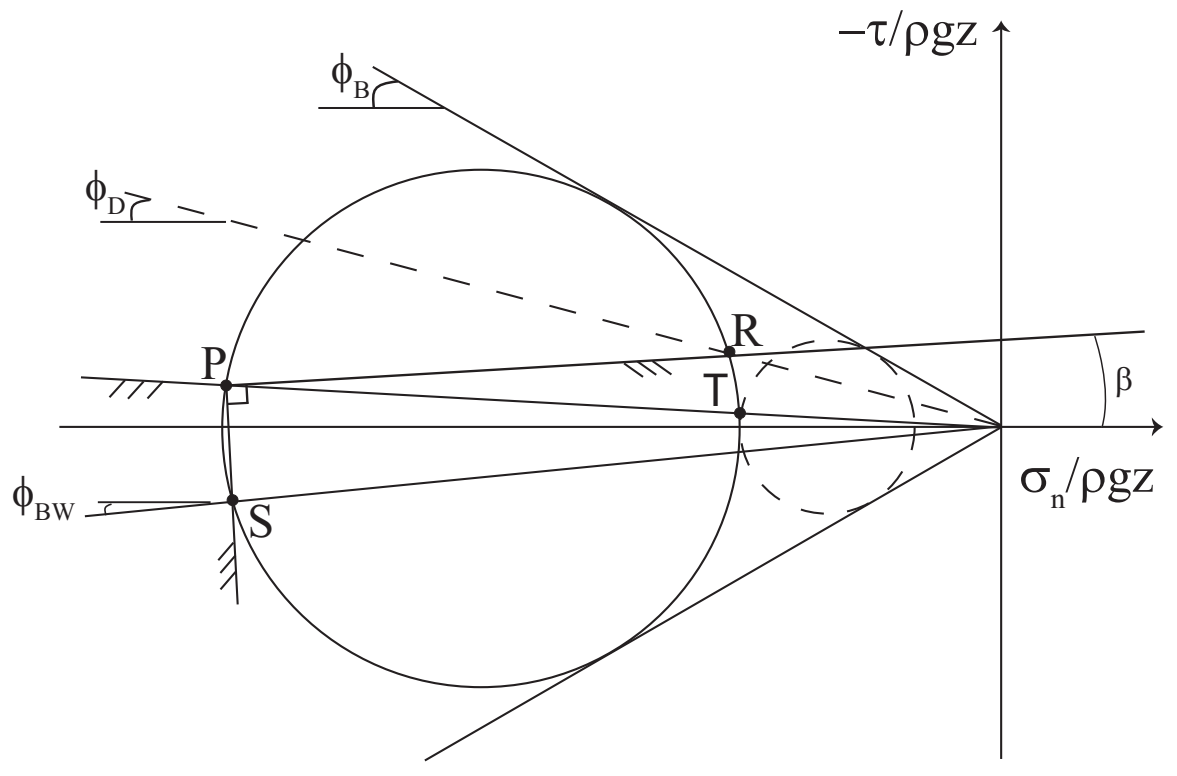

c)

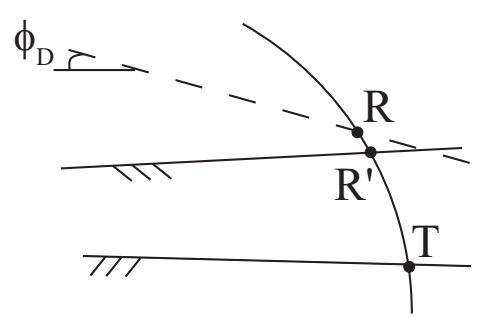

Figure 13: The Mohr's construction for the triangular wedge in a). The stress vector on facette 1, parallel to the topography, defines point $\mathrm{T}$ as well as the position of the pole on the circle, point $\mathrm{P}, \mathrm{b})$. The segment $\mathrm{PR}$ ' is dipping at $\beta$ and provides the stress acting on the décollement, point $\mathbf{R}^{\prime}$. The stress vector acting on facette 2 in a), is the point $S$ in $b$ ). The zoom in $c$ ) on the region of points $R$ and $T$ in $b$ ), shows that the décollement is not activated since $R^{\prime}$ requires a friction angle on the dćollement smaller than the value used for the construction. 


\subsection{Comparison with analogue experiments}

a)

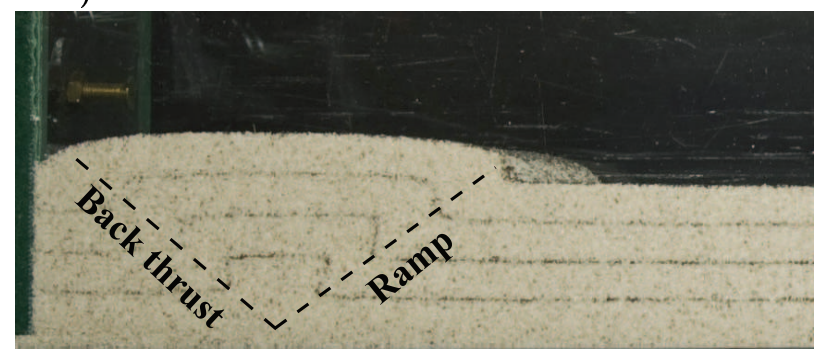

b)

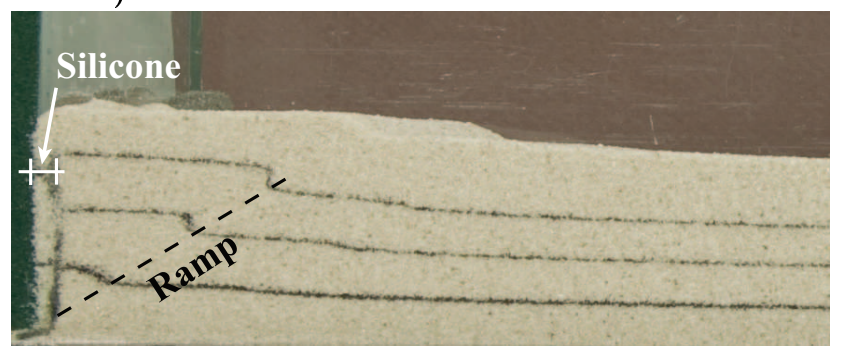

Figure 14: Two pictures of the laboratory experiments from the side showing a ramp and back-thrust for failure mode a) and a ramp only in b) in the absence and the presence of a layer of silicone along the back wall, respectively.

It is now proposed to compare the failure modes predicted from the virtual velocity field with the results of laboratory experiments with sand. The box is composed of two parallel side glass walls, separated by the distance of $7 \mathrm{~cm}$, clamped on a flat plate defining the plane of the décollement. The fourth plate corresponds to the back wall which exerts the force necessary to compress the granular, analogue material and can be displaced between the two lateral walls. The fifth plate is clamped on the décollement, and parallel to the back wall at a distance $D=37 \mathrm{~cm}$, initially. The internal region is filled with a well-sorted (good distribution in grain size) Fontainebleau quartz sand (median grain size of $250 \mu \mathrm{m}$ ). Its friction angle is of the order of $30^{\circ}$ (Schreurs et al., 2006). The friction over the side glass and the bottom plate is close to $15^{\circ}$ thanks to a proper treatment with chemical product. The friction over the back wall is much larger unless a thin layer of silicon putty (Dow Corning SGM 36) is inserted. This material has a viscosity of $\mu=5 \times 10^{4} \mathrm{Pas}$. The rate of compression is set to $8.3 \times 10^{-6} \mathrm{~m} / \mathrm{s}$ and the ramp in the sand inclined at $30^{\circ}$, so that the hanging wall is moving up at $4.8 \times 10^{-6} \mathrm{~m} / \mathrm{s}$. The silicone layer of thickness $2.5 \mathrm{~mm}$, if assumed to sustain a simple shear deformation, is flowing at a strain rate of $\dot{\gamma}=1.9 \times 10^{-3} 1 / \mathrm{s}$. The shear stress on the back wall is then $\tau=\dot{\gamma} \mu=96 P a$. The compressive force magnitude measured in the laboratory is of the order of $36 \mathrm{~N} / \mathrm{m}$ (per unit width), so that the normal stress on the back wall is on average $1800 \mathrm{~Pa}$. The equivalent friction coefficient is then $\tau / \sigma_{n}=5 \times 10^{-2}$ corresponding to the friction angle of $3^{\circ}$ which is indeed below the critical value $\phi_{B W}^{*}=6^{\circ}$ found above.

The initial set-up corresponds to a sand pack with $\alpha=\beta=0$ and a layer thickness of $20 \mathrm{~mm}$. The sand is deposited with a sand distributor, to ensure experimental reproducibility, by layers of up to $5 \mathrm{~mm}$ and separated by fine layers of colored sand acting as markers. The complete description of the experimental set up and of the protocol is presented by Cubas and al. (2009) and Souloumiac (2009). The results after shortening by $9 \mathrm{~mm}$ approximately are presented in Figure 14a and $\mathrm{b}$ in the absence and the presence of silicon putty on the back wall, respectively. The pictures are taken from the right side and present, through the side wall, the failure mode. It consists of a ramp and a back thrust, dashed segments, with a slight relief, Figure 14a. The back thrust is outcropping close to the back wall. In the presence of the silicone, a single discontinuity in the form of a ramp occurs rooting on the décollement at the back wall contact. Although many parameters are estimated to first order, these results validate the observation made in the previous section: the selection of the failure mode with or without back thrust is due to the friction over the back wall. The larger friction angles promote the presence of the back thrust. This interpretation will certainly contribute to the discussion initiated during the experimental benchmark of Schreurs et al. (2006). 


\section{Conclusion}

The objective was to propose a methodology which could ultimately permit to study systematically the $3 \mathrm{D}$ variations of the failure mode within accretionary wedges.

It is not the primal problem, constructed directly from the kinematics approach of the limit analysis, but its dual version which is found most convenient in view of the complexity of most strength criteria and the difficulty to account properly of the constraints (inequalities) due to their support functions. The dual variables are stress-like quantities, once appropriately scaled, although their distribution is not a-priori statically admissible. It is shown nevertheless, in the case of the 2D triangular wedge, that the dual stress field corresponds to the expected theoretical solution which satisfies equilibrium and is independent of the position along the topography and function only of the distance to this flat surface. The primal variables provide the virtual velocity field which characterizes the failure mode of the structure. The ramp and back thrust system defining the 2D failure mode is expressed as narrow strips of localized virtual deformation. This localization of virtual strain as well as the activation of the décollement are dilatant. This fact is known from the primal problem since dilatancy is required for the support function to be finite. It is interesting to recognize that the solution of the dual problem comes to the same conclusion. The back stop and the hanging wall are regions sustaining virtual rigid motions. The position of the failure mechanism to the front (super-critical) or the rear (sub-critical) of the wedge depends on the topographic slope, the décollement friction and the bulk friction angle. The exact relation (Dahlen, 1984; Lehner 1986) defines the critical topographic slope $\alpha_{c}$ which is captured exactly by the numerical procedure. It is further shown that the failure mode at the rear for sub-critical conditions $\left(\alpha<\alpha_{c}\right)$ could collapse to a single ramp rooting at the intersection of the back wall and the décollement instead of a ramp and back thrust system. The back wall friction angle predicted by a Mohr construction is, within graphical error, equal to the angle found numerically at the transition between these two modes of failure. Laboratory experiments, with sand and with or without silicone along the back wall, validate this finding quantitatively.

Two 3D examples are presented in the companion paper (Souloumiac et al., 2009). The first of the two examples has for objective to shed light on the validity of the $2 \mathrm{D}$ stability criterion in the presence of a lateral topographic slope variation (perpendicular to the direction of compression). It is shown that a lateral variation by $\pm 0.5^{\circ}$ from the critical $\alpha_{c}$, chosen in the central cross section, could prevent the deformation front to be at toe of the wedge in the $2 \mathrm{D}$ super-critical region. The $3 \mathrm{D}$ failure mechanism is then characterized by a ramp in the $2 \mathrm{D}$ sub-critical region which becomes diffuse with a decreasing dip as one move towards the 2D super-critical region. The second of the 3D examples is proposed to question the influence of the lateral wall friction on the failure mode produced typically in the laboratory. For a lateral wall friction of $15^{\circ}$ and a box width to length ratio of one, $20 \%$ of the width set up is affected by the lateral wall. The critical slope angle $\alpha_{c}=3.38^{\circ}$ is increased by as much as $1.5^{\circ}$. It is indeed necessary to increase significantly the weight of the wedge before super-critical conditions are met. The 2D stability are thus questioned and a 3D criterion based on the percentage of the surface of décollement which is activated is tentatively proposed and compared with predictions obtained with sand in the laboratory.

\section{Appendix A: Linear programming duality}

The objective of this appendix is to present the dualisation argument, which is classical in linear programming, with a notation consistent with the development proposed in this paper. 


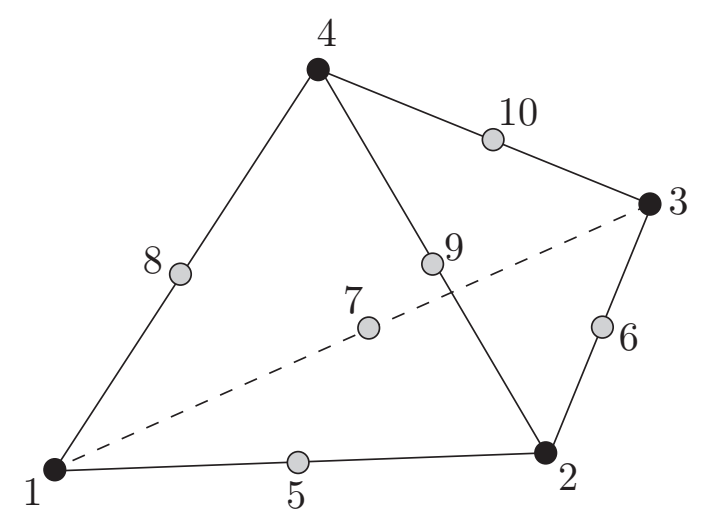

Figure 15: Quadratic tetrahedron element for 3D upper bound analysis.

This material is certainly not new and proposed here only for sake of completeness.

The primal problem is the following minimization search

$$
\begin{array}{cl}
\text { minimize } & { }^{t}\{c\}\{x\} \\
\text { subject to } & {[A]\{x\}=\{b\},} \\
& \{x\} \geq\{0\},
\end{array}
$$

in which the vector $\{x\}$ is the collection of $m$ unknowns and where there are $n$ equalities to be satisfied (the matrix $[A]$ is $n \times m$; the variables and dimension names differ from the ones defined in the main text). The dual problem is stated as

$$
\begin{array}{ll}
\text { Maximize } & { }^{t}\{b\}\{y\} \\
\text { subject to } & { }^{t}[A]\{y\}+\{s\}=\{c\}, \\
& \{s\} \geq\{0\},
\end{array}
$$

in terms of the $n$ unknowns in the vector $\{y\}$ and the additional $m$ slack variables in the vector $\{s\}$. The optimal solutions, in terms of the objective function, are the same for the two problems as it can be shown by computing the duality gap

$$
\text { gap } \equiv{ }^{t}\{c\}\{x\}-{ }^{t}\{b\}\{y\}={ }^{t}\{s\}\{x\} \geq 0 .
$$

The gap is always positive or nul and only nul if the two systems of equalities in (36) and (37) are satisfied. In that instance, the orthogonality condition ${ }^{t}\{s\}\{x\}=0$ applies. It is the dual problem (37) which is used for all examples in section 3 whereas the primal problem (36) was set up in section 2 from the maximum strength theorem with a spatial discretisation for the velocities and the deformation components $\bar{\lambda}$.

\section{Appendix B: Generalisation to 3D}

The 3D discretization is constructed with ten-node tetrahedra, as illustrated in Figure 15. The mid-side nodes are located at equal distances between the vertex nodes and all sides are planar surfaces. The pseudo-equilibrium matrix analogous to (25) is given by 
$[\overline{\mathcal{B}}]^{e}=-\frac{1}{24}\left[\begin{array}{cccccccccc}3 \mathrm{P}_{1} & -\mathrm{P}_{2} & -\mathrm{P}_{3} & -\mathrm{P}_{4} & 4 \mathrm{P}_{2} & 0 & 4 \mathrm{P}_{3} & 4 \mathrm{P}_{4} & 0 & 0 \\ -\mathrm{P}_{1} & 3 \mathrm{P}_{2} & -\mathrm{P}_{3} & -\mathrm{P}_{4} & 4 \mathrm{P}_{1} & 4 \mathrm{P}_{3} & 0 & 0 & 4 \mathrm{P}_{4} & 0 \\ -\mathrm{P}_{1} & -\mathrm{P}_{2} & 3 \mathrm{P}_{3} & -\mathrm{P}_{4} & 0 & 4 \mathrm{P}_{2} & 4 \mathrm{P}_{1} & 0 & 0 & 4 \mathrm{P}_{4} \\ -\mathrm{P}_{1} & -\mathrm{P}_{2} & -\mathrm{P}_{3} & 3 \mathrm{P}_{4} & 0 & 0 & 0 & 4 \mathrm{P}_{1} & 4 \mathrm{P}_{2} & 4 \mathrm{P}_{3}\end{array}\right]$,

where

$$
{ }^{t} \mathrm{P}_{i}=2 A_{i}\left[\begin{array}{cccccc}
n_{1}^{i} & 0 & 0 & n_{2}^{i} & n_{3}^{i} & 0 \\
0 & n_{2}^{i} & 0 & n_{1}^{i} & 0 & n_{3}^{i} \\
0 & 0 & n_{3}^{i} & 0 & n_{1}^{i} & n_{2}^{i}
\end{array}\right],
$$

with $\left(n_{x}^{i}, n_{y}^{i}, n_{z}^{i}\right)$ being the unit outward normal to face $i$ (opposite node $i$ ) and $A_{i}$ the area of that face.

The pre-defined discontinuities in 3D are constructed by patches of zero-thickness and composed of three tetrahedra, as shown in Figure 16. Again, this patch of tetrahedra is treated in the same way as the regular bulk elements and the fact that the element volume is equal to zero does not pose any difficulty. Also, similar to 2D discontinuities, the internal discontinuity nodes are eliminated by making appropriate assumptions about the variation of the velocities across the discontinuity.

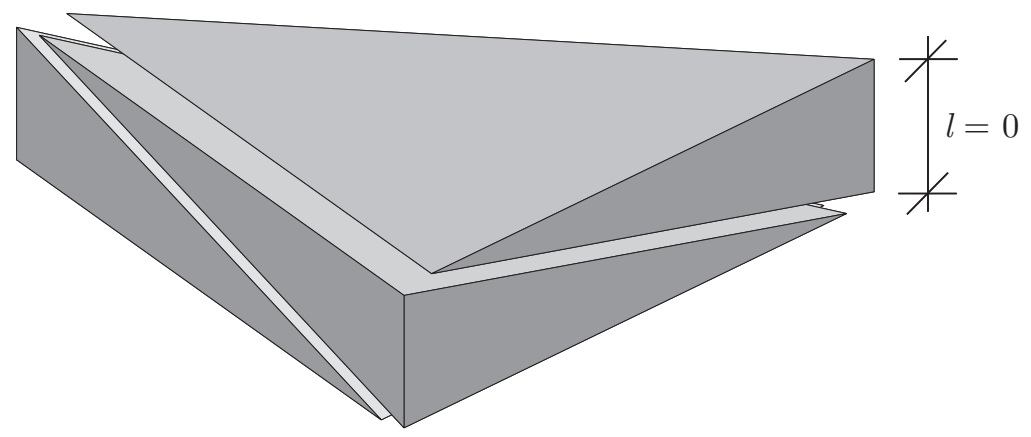

Figure 16: Discontinuity patch consisting of three zero-thickness tetrahedra for constructing a discontinuity of known geometry and zero thickness in $3 \mathrm{D}$.

\section{Appendix C: Second-order cone programming}

This last Appendix presents the conversion of the general non-linear upper bound limit analysis problem (32) into second-order cone programming (SOCP) format. The most common of these formats comes in the form of the following generalization of the primal LP problem (36):

$$
\begin{array}{cl}
\text { minimize } & { }^{t}\{c\}\{x\} \\
\text { subject to } & {[A]\{x\}=\{b\},} \\
& \{x\}_{i} \in \mathcal{K}_{i}, i=1, \ldots, n,
\end{array}
$$

where the total solution vector $\{x\}$ is assumed to be partitioned into $n$ subvectors $\{x\}_{i}$. For each of these subvectors a conic inequality constraint, given by the last line in (40), is imposed. The mathematical definitions of what constitutes a cone are relatively stringent and cannot easily be circumvented (see, e.g., Ben-Tal and Nemirovski 2001, for details). However, for the present 
application it suffices to know that the Drucker-Prager criterion, by a suitable transformation of variables, is cast in the following quadratic cone:

$$
\mathcal{K}=\left\{\{x\} \in \mathbb{R}^{\mathrm{m}+1} \mid \mathrm{x}_{1} \geq \sqrt{\sum_{\mathrm{j}=2}^{\mathrm{m}+1} \mathrm{x}_{\mathrm{j}}^{2}}\right\}
$$

This transformation is achieved by introducing a new set of variables $\{\rho\}$ :

$$
\{\rho\}=[D]\{\sigma\}+\{d\},
$$

where

$$
[D]=\left[\begin{array}{rrrrrr}
-\alpha_{D P} & -\alpha_{D P} & -\alpha_{D P} & & & \\
1 / \sqrt{6} & -1 / \sqrt{6} & & & & \\
& 1 / \sqrt{6} & -1 / \sqrt{6} & & \\
-1 / \sqrt{6} & & 1 / \sqrt{6} & & & \\
& & & 1 & & \\
& & & & 1 & \\
& & & & & 1
\end{array}\right], \quad\{d\}=\left\{\begin{array}{c}
C_{D P} \\
0 \\
0 \\
0 \\
0 \\
0 \\
0
\end{array}\right\} .
$$

The Drucker-Prager criterion (5) can then be written as

$$
\mathcal{K}_{D P}=\left\{\{\rho\} \in \mathbb{R}^{7} \mid \rho_{1} \geq \sqrt{\sum_{\mathrm{j}=2}^{7} \rho_{\mathrm{j}}^{2}}\right\},
$$

which is a quadratic cone. Hence, in the case where the yield criterion is of the Drucker-Prager type, the SOCP standard form of the general nonlinear upper bound limit analysis problem (32) reads

$$
\begin{array}{ll}
\text { maximize } & \alpha_{U} \\
\text { subject to } \quad & { }^{t}[\overline{\mathcal{B}}]\{\tilde{\sigma}\}={ }^{t}[H]\{\tilde{R}\}+\alpha_{U}\left\{T_{0}\right\}+\{G\} \\
& \{\rho\}=[D]\{\tilde{\sigma}\}+\{d\} \\
& \{\rho\}_{i} \in \mathcal{K}_{D P} \text { for } i=1 \text { to } 3, \text { for each element in } \Omega^{h} .
\end{array}
$$

This problem is solved using the general purpose SOCP solver MOSEK (2008). In some cases it is possible to eliminate the physical stress variables $\{\tilde{\sigma}\}$ to end up with only problem unknowns $\{\rho\}$ and $\left\{\alpha_{U}\right\}$ (see Krabbenhoft et al., 2007 for details). This is exploited for problems where $\alpha_{D P}$ is non-zero which implies that ${ }^{t}[D][D]$ is non-singular and the physical stress variables are expressed entirely in terms of $\{\rho\}$. 


\section{References}

[] Anderheggen, E., and H. Knöpfel (1972), Finite element limit analysis using linear programming, International Journal of Solids and Structures, 8, 1413-1431.

[l Ben-Tal, A. and Y. Nemirovski (2001), Lectures in Modern Convex Optimization: Analysis, Algorithms and Engineering Applications, MPS-SIAM Series on Optimization.

[l Bottero, A., Negre, R., Pastor, J. and S. Turgeman (1980), Finite element method and limit analysis theory for soil mechanics problems, Computer Methods in Applied Mechanics and Engineering, 22, 131-149.

[] Cubas, N., Y.M. Leroy, and B. Maillot (2008), Prediction of thrusting sequences in accretionary wedges, J. Geophys. Res., J. Geophys. Res., 113, B12412, doi:10.1029/2008JB005717.

[] Cubas, N., B. Maillot and C. Barnes (2009), Statistics of the experimental growth of a sand wedge, submitted for publication.

[] Davis R.O. and A.P. Selvadurai (2002), Plasticity and geomechanics, Cambridge University Press.

[] Dahlen, F.A. (1984), Noncohesive critical Coulomb wedges : an exact solution, J. Geophys. Res., 89, B12, 10125-10133.

[] Day, R.A. and D.M. Potts (1994) Zero thickness interface elements - numerical stability and application, Int. J. for Numerical and Analytical Methods in Geomechanics, 18, 689-708.

[] Krabbenhøft, K., and L. Damkilde (2003), A general nonlinear optimization algorithm for lower bound limit analysis, International Journal for Numerical Methods in Engineering, 56, $165-184$.

[] Krabbenhøft, K., A.V. Lyamin, M. Hjiaj, and S.W. Sloan (2005) A new discontinuous upper bound limit analysis formulation, Int. J. Num. Meth. Eng., 63, 1069-1088.

[l Krabbenhøft, K., Lyamin, A.V., and S. W. Sloan (2007), Formulation and solution of some plasticity problems as conic programs, International Jounal of Solids and Structures, 44, $1533-1549$.

[l Krabbenhøft, K., Lyamin, A. V. and S. W. Sloan (2008) Three-dimensional Mohr-Coulomb limit analysis using semidefinite programming, Comunications in Numerical Methods in Engineering, 24, 1107-1119.

[l Krabbenhøft, K., A.V. Lyamin, M. Hjiaj, and S.W. Sloan (2005) A new discontinuous upper bound limit analysis formulation, Int. J. Num. Meth. Eng., 63, 1069-1088.

[] Krabbenhøft, K., A.V. Lyamin and S.W. Sloan (2007), Formulation and solution of some plasticity problems as conic programs, Int. J. Solids and Structures, 44, 1533-1549.

[] Lehner, F.K. (1986), Comments on "Noncohesive Critical Coulomb Wedges : An Exact Solution", J. Geophys. Res., 91, B1, 793-796.

[] Lyamin, A. V., and S. W. Sloan (2002), Upper bound limit analysis using linear finite elements and non-linear programming, International Journal for Numerical and Analytical Methods in Geomechanics, 26, 181-216. 
[] Maillot, B. and Y.M. Leroy (2003), Optimal dip based on dissipation of back thrusts and hinges in fold-and-thrust belts, J. Geophys. Res., 108, B6, 2320-2336.

[] Maillot, B. and Y.M. Leroy (2006), Kink-fold onset and development based on the maximum strength theorem, J. Mech. Phys. Solids, 54, 2030-2059.

[] Mandl, G. (2005), Rock joints, the mechanical genesis, Springer.

[] MOSEK (2008), optimization software, <http://www.mosek.com>.

[] Pastor, J. (1978), Limit analysis: numerical determination of complete statical solutions. Application to the vertical cut, (in French) Journal de Mécanique appliquée, 2, 168-196.

[l Pastor, J. (2006), Personal communication.

[l Pastor, F., Ph. Thore, E. Loute, J. Pastor, M. Trillat (2008), Convex optimization and limit analysis: Application to Gurson and porous Drucker-Prager materials, Eng. Fracture Mech., $75,1367-1383$.

[l Salençon, J. (1974), Théorie de la plasticité pour les applications à la mécanique des sols, edited by Eyrolles, Paris, (English translation : Applications of the theory of plasticity in soil mechanics, John Wiley \& Sons Ltd, Chichester, 1977).

[l Salençon, J. (2002), De l'élasto-plasticité au calcul à la rupture, Editions École Polytechnique, Palaiseau, and Ellipses, Paris.

[l SARPP (2008), Structural Analysis and Rock Physics Program, Finite-element and limitanalysis general program, Ecole Normale Supérieure, Paris.

[] Schreurs, G., S.J.H. Buiter, D. Boutelier, G. Corti, E. Costa, A.R. Cruden, J.-M. Daniel, S. Hoth, H.A. Koyi , N. Kukowski, J. Lohrmann, A. Ravaglia, R.W. Schlische, M. Oliver Withjack, Y. Yamada, C. Cavozzi, C. Delventisette, J.A. Elder Brady, A. Hoffmann-Rothe, J.M. Mengus, D. Montanari, F. Nilforoushan (2006), Analogue benchmarks of shortening and extension experiments, in Analogue and numerical modelling of crustal-scale processes, edited by S.J.H. Buiter and G. Schreurs, pp. 1-27, London Geol. Soc. Spec. Publ.

[l Sciamanna, S., W. Sassi, R. Gambini, J.L. Rudkiewicz, F. Mosca and C. Nicolai (2004), Predicting hydrocarbon generation and expulsion in the Southern Apennines Thrust Belt by 2D Integrated Structural and Geochemical Modeling: Part I - Structural and thermal evolution, in Deformation, fluid flow, and reservoir appraisal in foreland fold and thrust belts, edited by R. Swennen, F. Roure, J.W. Granath, pp.51-67, A.A.P.G. Heldberg series, 1.

[] Sloan, S. W. (1989), Upper bound Limit Analysis using Finite Elements and Linear Programming, International Journal for Numerical and Analytical Methods in Geomechanics, 13, 263-282.

[] Sloan, S.W. and P.W. Kleeman (1995), Upper bound limit analysis using discontinuous velocity fields, Comp. Meth. Appl. Mech. Engrg., 127, 293-314.

[] Souloumiac, P., Y.M. Leroy, K. Krabbenhøft K. and B. Maillot (2007), Predicting stress in fault-bend fold by optimization, submitted for publication.

[] Souloumiac, P., K. Krabbenhøft, Y.M. Leroy, B. Maillot (2009) Failure in accretionary wedges with the maximum strength theorem: 3D applications, submitted for publication. 
[] Zoetemeijer, R. and W. Sassi (1992) 2D reconstruction of thrust evolution using the faultbend fold method, in Thrust tectonics, edited by K. McClay, pp.133-140, London Geol. Soc. Spec. Publ. 